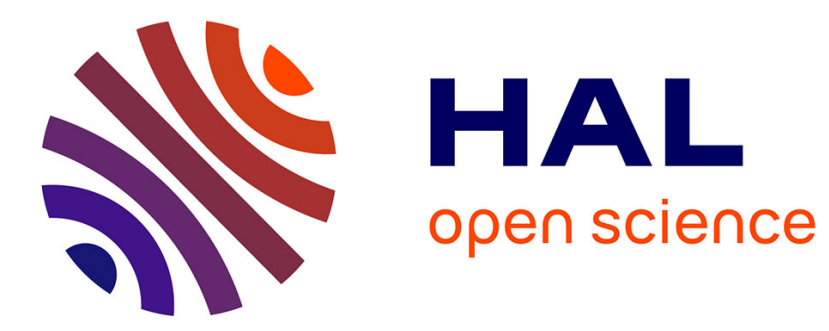

\title{
Mathematical analysis and solution methodology for an inverse spectral problem arising in the design of optical waveguides
}

Hélène Barucq, Chokri Bekkey, Rabia Djellouli

\section{- To cite this version:}

Hélène Barucq, Chokri Bekkey, Rabia Djellouli. Mathematical analysis and solution methodology for an inverse spectral problem arising in the design of optical waveguides. Inverse Problems in Science and Engineering, In press. hal-01807247

\section{HAL Id: hal-01807247 \\ https://hal.science/hal-01807247}

Submitted on 4 Jun 2018

HAL is a multi-disciplinary open access archive for the deposit and dissemination of scientific research documents, whether they are published or not. The documents may come from teaching and research institutions in France or abroad, or from public or private research centers.
L'archive ouverte pluridisciplinaire HAL, est destinée au dépôt et à la diffusion de documents scientifiques de niveau recherche, publiés ou non, émanant des établissements d'enseignement et de recherche français ou étrangers, des laboratoires publics ou privés. 


\title{
Mathematical analysis and solution methodology for an inverse spectral problem arising in the design of optical waveguides.
}

Hélène Barucq ${ }^{1}$, Chokri Bekkey, ${ }^{2}$ and Rabia Djellouli*3

${ }^{1}$ INRIA Bordeaux Sud-Ouest Research Center, Project-Team Magique-3D, LMA-UMR CNRS 5142, Université de Pau et des Pays de l'Adour, Avenue de l'Université, BP 1155, 64013 PAU Cedex, FRANCE.

${ }^{2}$ Laboratoire BIMS, Institut Pasteur de Tunis \& Département de Mathématique, Faculté des Sciences, Université de Monastir, TUNISIA.

${ }^{3}$ Interdisciplinary Research Institute for the Sciences (IRIS), Department of Mathematics, California State University, Northridge \& INRIA Associate Team Magic, 18111 Nordhoff Street, CA 91330 Northridge, USA.

April 19, 2018

\begin{abstract}
We analyze mathematically the problem of determining refractive index profiles from some desired/measured guided waves propagating in optical fibers. We establish the uniqueness of the solution of this inverse spectral problem assuming that only one guided mode is known. We then propose an iterative computational procedure for solving numerically the considered inverse spectral problem. Numerical results are presented to illustrate the potential of
\end{abstract}

*corresponding author: rabia.djellouli@csun.edu 
the proposed regularized Newton algorithm to efficiently and accurately retrieve the refractive index profiles even when the guided mode measurements are highly noisy.

\section{Introduction}

Inverse spectral problems (ISP) are a class of problems that is relevant to a wide range of applications in science and technology. Examples of such applications include large static structures such as buildings and bridges, as well as smaller dynamic structures such as automobiles and helicopters. These structures require extensive vibration testing and analysis during the design and the development stages. The determination of the structural parameters is one of the most important stages in the analysis. This is accomplished by solving ISPs to calculate coefficients of the differential systems corresponding to the considered mathematical models. The determination of the variations of the density of the earth from its eigenfrequencies is another example of an ISP arising in the geophysical science field. All these important applications require at some point of their studies the solution of ISPs. For this reason, ISPs received during the past three decades a great deal of attention by applied mathematicians and engineers, as demonstrated by the prolificness of literature and conferences dedicated to this topic.

Inverse spectral problems can be broadly divided into two categories: inverse spectral domain problems (ISDP) and inverse spectral parameter problems (ISPP). In the first category, i.e., the inverse spectral domain problems (ISDP), the goal is to find the shape of a region from the partial or total knowledge of the spectrum of an elliptic operator, such as Laplace operator. Although the first ISDP was formulated in 1882 by Sir A. Shuster, who introduced spectroscopy as a way to find a shape of a bell by means of the sounds which it is capable of sending out, no significant progress was accomplished in this area until the mid 1960's. Indeed, the publication of the fundamental paper by Kac in 1966 set the stage for the subsequent mathematical and numerical investigations of this category of problems (see, for example, the short review by Protter [1] and the book of Bérard [2]). Despite the publication of several works on the mathematical and numerical analysis of ISDP's (see [3]-[5], among others), there are still many open questions [6].

In the second category, the inverse spectral parameter problems (ISPP), the aim is to recover material properties from the a priori knowledge of the natural frequencies or mode shape measurements. Hence, this class of problems consists in identifying parameters of differential operators from their corresponding spectrum. One of the fundamental papers addressing the mathe- 
matical aspects of ISPPs was authored by Borg, who analyzed the particular case of the Sturm-Liouville operator [7], which is primarily a one-dimensional model problem. It amounts to determining the density of a vibrating string from its fundamental tone and overtone. Since then, this model problem has been extensively studied. Indeed, various proofs on the uniqueness have been given in [8]-[11] and constructive methods have been suggested in [12][18]. Nowadays, an ISPP for the Sturm-Liouville operator is considered to be relatively well understood [19]-[20] and some of the results pertaining to the construction of the solution, as well as its uniqueness, have been extended to more general one-dimensional operators [21]-[22]. For multidimensional ISPPs, the situation is more complicated despite the important efforts made and documented in literature (see [23]-[29], among others). Specifically, for two-dimensional ISPPs, the most significant results have been established when the spectrum data are given for rectangular-shaped and bounded membranes [30]-[33]. ISPP for the linear Schrödinger operator in the case of unbounded domains was first analyzed in [34]. However, this study is restricted to the case where the sought-after potentials are periodic. We must point out that most of the recent studies provide only theoretical results via different mathematical approaches (see, e. g., [35]-[43]).

The main goal of this paper is to study from both mathematical and numerical viewpoints the problem of determining refractive index profiles from some measured or desired guided waves propagating in optical fibers. This ISPP occurs in network communications when developing optical waveguides that achieve a desired lightwave transmission. Extensive effort has been deployed in the last two decades to solve numerically this class of inverse spectral problems, as attested by the numerous publications (see, e.g., [44]-[54]). Prior to that, an interesting attempt was made in the mid $70^{\prime} s$ [55]. The proposed approach for computing the refractive index profile uses the measurements collected from the near field intensity distribution. This method seems to suffer from the presence of spurious modes. The more recent work presented in [53] proposes a computational procedure to retrieve the refractive index profile of multi-layered circular optical fibers from the knowledge of the propagation constants and their corresponding eigenwaves under the weak guidance propagation assumption. This work extends the ideas and techniques developed in [47] and [51] in the case of a one-layered optical fiber. The proposed numerical method employs a functional cost built from the characteristic equation that connects the refractive indices of the waveguide's layers to the propagation constants of its eigenwaves. It is defined as the determinant of the matrix giving the coefficients of the wavefield decomposed over a basis of Hankel functions. This approach seems to be limited to circular-shaped waveguides due to the choice of the basis functions. For planar multi-layered 
waveguides, the waveguide spectroscopy method is used in [44]. This method consists of minimizing the distance between the computed and the measured propagation constants vectors. The computed ones are obtained as the roots of well-known characteristic equations. The application of this method has been extended to waveguides with piecewise constant refractive index profiles and arbitrary cross-sectional boundaries [48], [50], [52], [53]. The authors propose a strategy that is based on the solution of a nonlinear nonself-adjoint eigenvalue problem corresponding to a system of weakly singular integral equations. The numerical approach introduced in [46] reconstructs the refractive index profile of a cylindrical waveguide from the knowledge of the corresponding near field in the case of Maxwell system. The main idea of this technique is to reformulate the problem into a set of one-dimensional problems after dividing the optical waveguide into homogeneous cylindrical layers of a prescribed thickness. The accuracy and effectiveness of the method are highly dependent on the thickness parameter values. The work presented in [54] seems very close to ours. However, the approach adopted in [54] differs from our solution methodology by several aspects, chief among them: (a) the ISPP is formulated in [54] as an optimization problem under constraints and is solved via the use of Lagrange multipliers, and (b) the Fréchét derivatives with respect to the refractive index profile are computed using a finite difference (FD) approximation of order 1 . Due to the constraints considered in [54], the method is limited to continuous profiles at the interface core-cladding and its efficiency (accuracy and convergence) is sensitive to the FD step size value when approximating the derivatives.

In the present work, we investigate the question of the uniqueness of the solution for the considered ISPP and characterize its Fréchet derivative with respect to the refractive index profile. At the numerical level, we propose a solution methodology that falls in the category of regularized iterative methods. The proposed computational procedure possesses the following four main features: (a) a computationally efficient usage of the exact sensitivities of the guided modes to the specified refractive index parameters, (b) the solution of only one eigenvalue problem at each Newton iteration, (c) a Tikhonov-like regularization to restore the stability, and (d) an efficient computational method coupling a local boundary condition to a finite element formulation for solving the direct eigenvalue problem. Note that the mathematical difficulties, computational issues, and solution approaches addressed in this paper are relevant to many ISPs arising in other applications. The remainder of this paper is organized as follows. In Section 2, we recall the forward eigenvalue problem that characterizes the propagation of guided modes in a homogeneous optical fiber. We assume the propagation to be under the weak guidance conditions [56]-[58]. In Section 3, we state the ISPP 
of interest and establish mathematical results pertaining to (a) the uniqueness of the solution, and (b) the Fréchet derivative of the eigenmodes with respect to the refractive index profile. Section 4 is devoted to the description of the proposed solution methodology for solving the considered ISPP. We present in Section 5 various illustrative numerical results to highlight the performance efficiency of the proposed computational procedure. Concluding remarks are included in Section 6.

\section{The Direct Problem}

\subsection{Nomenclature and assumptions}

An optical fiber can be viewed as a cylindrical dielectric structure that is extended along its propagation axis, denoted here by $O x_{3}$ (see Figure 1(a)). In its transverse directions, $O x_{1}$ and $O x_{2}$, an optical fiber is constituted of two open subsets of $\mathbb{R}^{2}$ : a core region denoted by $\Omega$ and a cladding region $\Omega^{e}=\mathbb{R}^{2} \backslash \bar{\Omega}$ (see Figure $1(\mathrm{~b})$ ). The core $\Omega$ is assumed to be Lipschitz continuous. The cladding $\Omega^{e}$ is assumed to be infinitely extended since guided waves decrease exponentially to zero out of the core region and the radius of the cladding is in practice very large compared to the radius of fiber core [56]-[59]. The core and the cladding regions are fully determined once the refractive index profile $n$ of the considered fiber is known. The proposed study is limited to optical fibers with homogeneous cladding. Therefore, the class of refractive index profiles $n$ we consider here are positive real-valued functions depending on $\mathbf{x}=\left(x_{1}, x_{2}\right)$ such that $n \in L^{\infty}\left(\mathbb{R}^{2} ; \mathbb{R}_{+}^{\star}\right)$, and:

$$
\exists n_{\infty} \in \mathbb{R}_{+}^{*} \text { such that } n(\mathbf{x})=n_{\infty} \text { a.e. } \mathbf{x} \in \Omega^{e} .
$$

Furthermore, to ensure the guided waves propagation in the considered fiber, the refractive index profile must attain its maximum inside the core of the fiber [56]-[59], that is:

$$
\exists n_{+} \in \mathbb{R}_{+}^{\star} \quad \text { such that ess } \sup _{\mathbf{x} \in \mathbb{R}^{2}} n=n_{+}>n_{\infty},
$$

where ess sup denotes the essential supremium [59].

We consider the following class of refractive index profiles:

$$
\begin{aligned}
& \mathcal{N}=\left\{n \in L^{\infty}\left(\mathbb{R}^{2} ; \mathbb{R}_{+}^{\star}\right) ; \exists n_{\infty}>0\right. \\
&\left.n(\mathbf{x})=n_{\infty} \text { a.e. in } \Omega^{e} \text { and } \operatorname{ess} \sup _{\mathbf{x} \in \mathbb{R}^{2}} n=n_{+}>n_{\infty}\right\}
\end{aligned}
$$




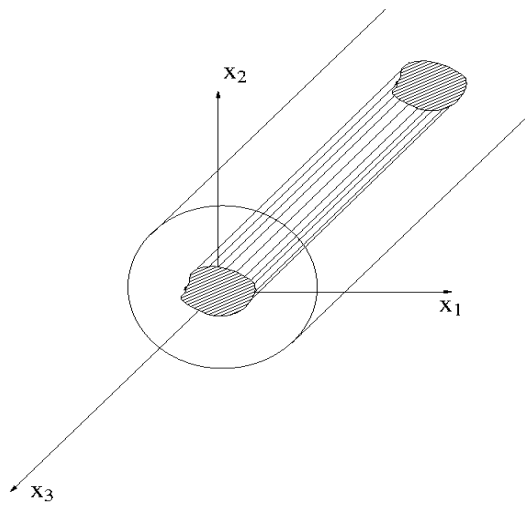

(a)

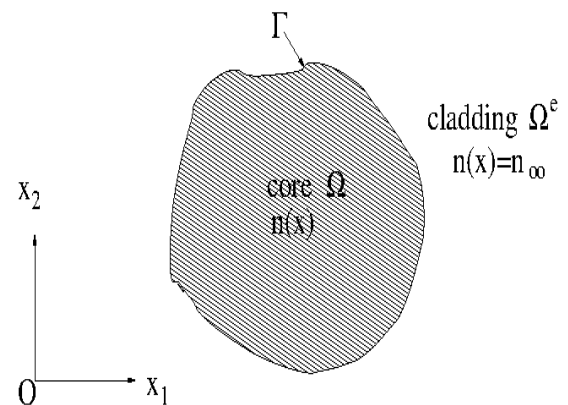

(b)

Figure 1: (a) An optical fiber, (b) a transverse section of a homogeneous optical fiber.

and the set of admissible refractive index profiles considered in this study, denoted by $\mathcal{N}_{1}$, is defined by:

$$
\mathcal{N}_{1}=\{n \in \mathcal{N} ; n \text { being piecewise continuous }\}
$$

Note that the set $\mathcal{N}_{1}$ encompasses all refractive index profiles encountered in practical applications [57]-[58], [60].

\subsection{Problem statement}

We consider the propagation of guided modes under the weak guidance conditions. Hence, we assume the refractive-index variations to be small compared to the wavenumber $k$. In this situation, the propagation of guided waves in homogeneous optical fibers can be formulated as the following scalar eigenvalue problem EVP [56]-[59]:

$$
(\mathbf{E V P})\left\{\begin{array}{cl}
\text { Find } \beta \in] k n_{\infty}, k n_{+}[\text {and } & u \in L^{2}\left(\mathbb{R}^{2}\right) ; u \neq 0 \text { such that: } \\
\Delta u+k^{2} n^{2} u=\beta^{2} u & \text { in } \mathbb{R}^{2} \\
{[u]=\left[\frac{\partial u}{\partial \nu}\right]=0} & \text { on } \Gamma=\partial \Omega
\end{array}\right.
$$

where:

- $\beta$ is the propagation constant. It represents the speed of the electromagnetic field along its propagation axis $O x_{3}$ (see Figure 1(a)).

- $u$ is the associated eigenfunction of $\beta$. It represents any transverse component of the electromagnetic field that propagates in the fiber. The couple $(\beta, u)$ is called a guided mode. 
- $n$ is an admissible refractive index, that is, $n \in \mathcal{N}_{1}$.

- The brackets [. . in equation (6) represent the jump across the corecladding interface $\Gamma$ and $\nu$ is the unit normal vector on $\Gamma$ oriented towards the cladding $\Omega^{e}$. The transmission conditions given by (6) express the continuity of the tangential components of the electromagnetic field over the core-cladding interface $\Gamma$ (see Figure $1(b)$ ).

EVP has been analyzed extensively, both mathematically and numerically. Results pertaining to the existence of guided modes, their number, as well as their sensitivities to the opto-geometrical parameters $k$ and $n$ of the considered waveguide can be found in [56]-[59], among other references.

\section{The Inverse Spectral Problem}

\subsection{Problem statement}

As stated earlier in the introduction section, our aim is to propose a solution methodology that efficiently retrieves the refractive index profile from the knowledge of some guided modes $(\beta, u)$ propagating, at a fixed frequency, in a given optical fiber. To formulate mathematically this inverse problem, we first recall that for a prescribed frequency, characterized by the wavenumber $k$, and a given refractive index $n \in \mathcal{N}_{1}$, EVP admits a finite number $S$ of propagation constants, counted with their multiplicities, denoted by $\boldsymbol{\beta}_{S}=\left(\beta_{1}, \cdots, \beta_{S}\right)^{T}$ and their corresponding eigenfunctions denoted by $\mathbf{u}_{S}=\left(u_{1}, \cdots, u_{S}\right)^{T}$ [59]. Therefore, EVP defines a vector-valued mapping $\mathbf{F}_{S}=\left(F_{1}, F_{2}, \cdots, F_{S}\right)^{T}$, such that:

$$
\mathbf{F}_{S}: n \longrightarrow \mathbf{F}_{S}(n)=\boldsymbol{\beta}_{S} .
$$

Note that the $l^{\text {th }}$ coordinate $F_{l}(n)=\beta_{l}$ is the propagation constant corresponding to the $l^{\text {th }}$ guided mode, counted with its multiplicity. This means that the $\beta_{l}$ s are not necessarily distinct. The considered inverse spectral problem can be then formulated as follows:

$($ ISPP $)\left\{\begin{array}{l}\text { Given the first } I \text { guided modes }\left(\beta_{1}, u_{1}\right),\left(\beta_{2}, u_{2}\right), \cdots,\left(\beta_{I}, u_{I}\right) \\ (1 \leq I \leq S) \text { propagating at a fixed frequency } k, \text { find the refrac- } \\ \text { tive index } n \in \mathcal{N}_{1} \text { such that: } \\ \qquad \mathbf{F}_{I}(n)=\boldsymbol{\beta}_{I}=\left(\beta_{1}, \beta_{2}, \cdots, \beta_{I}\right)^{T} .\end{array}\right.$ 
Remark. Note that the considered ISPP can also be extended to the case where the guided modes are measured for multiple frequencies corresponding to $N_{F}$ wavenumbers $k_{1}, k_{2}, \cdots, k_{N_{F}}$. For each considered wavenumber $k_{l}\left(1 \leq l \leq N_{F}\right)$, its corresponding first $I_{l}$ propagation constants $\boldsymbol{\beta}_{I_{l}}=$ $\left(\beta_{1_{l}}, \beta_{2_{l}}, \cdots, \beta_{I_{l}}\right)^{T}$ and their associated eigenfunctions $\mathbf{u}_{I_{l}}=\left(u_{1_{l}}, u_{2_{l}}, \cdots, u_{I_{l}}\right)^{T}$ are given respectively in ISPP. Therefore, the number of eigenmodes $I$ in ISPP becomes $I=\sum_{l=1}^{N_{F}} I_{l}$.

\subsection{Mathematical results}

The first result establishes the uniqueness of the solution of ISPP.

Proposition 3.1. The refractive index $n$, solution of ISPP, can be uniquely determined in $\mathcal{N}_{1}$ from only the knowledge of the fundamental mode $\left(\beta_{1}, u_{1}\right)$.

Proof. Consider ISPP with $I=1$, that is the fundamental eigenmode pair $\left(\beta_{1}, u_{1}\right)$ is given. Let $n_{1}$ and $n_{2}$ be two solutions of ISPP. Then, equation (5) implies:

$$
\Delta u_{1}+k^{2} n_{l}^{2} u_{1}=\beta_{1}^{2} u_{1} \quad \text { in } \mathbb{R}^{2} \text { with } \quad n_{l}=n_{l, \infty}>0 \text { in } \Omega^{e} ; l=1,2 .
$$

It follows from the difference between the two equations given by (9) that:

$$
\left(n_{1}^{2}-n_{2}^{2}\right) u_{1}=0 \quad \text { a.e. in } \mathbb{R}^{2}
$$

In particular, we have:

$$
\left(n_{1, \infty}-n_{2, \infty}\right) u_{1}=0 \quad \text { a.e. in } \Omega^{e},
$$

Hence, either $u_{1}=0$ a.e. in $\Omega^{e}$ or $n_{1, \infty}=n_{2, \infty}$.

Case1: Assume that $u_{1}=0$ a.e. in $\Omega^{e}$.

Since $u_{1} \in H^{2}\left(\mathbb{R}^{2}\right)$ [59], then we have:

$$
u_{1}=\frac{\partial u_{1}}{\partial \nu}=0 \quad \text { a.e. on } \partial \Omega^{e}
$$

and the standard trace theorems can be applied to $u_{1}$ on the boundary for both Dirichlet and Neumann traces. It follows that $u_{1}$ satisfies the following homogeneous boundary value problem:

$$
\left\{\begin{array}{cc}
\Delta u_{1}+\left(k^{2} n_{1}^{2}-\beta_{1}^{2}\right) u_{1}=0 & \text { in } \Omega \\
u_{1}=\frac{\partial u_{1}}{\partial \nu}=0 & \text { on } \partial \Omega
\end{array}\right.
$$


Hence, using the unique continuation principle (see, e.g., [61]-[62]), we deduce that:

$$
u_{1}=0 \quad \text { a.e. in } \Omega \text {, }
$$

and therefore

$$
u_{1}=0 \quad \text { a.e. in } \mathbb{R}^{2},
$$

which contradicts $u_{1}$ being an eigenfunction, i.e., $\left\|u_{1}\right\|_{2} \neq 0$.

Case2: Assume that $n_{1, \infty}=n_{2, \infty}$.

If $n_{1} \neq n_{2}$, then, since the refractive index is a positive valued function, there must be an open subset $D \subseteq \Omega$, such that:

$$
n_{1}^{2}-n_{2}^{2} \neq 0 \quad \text { a.e. in } D \text {. }
$$

It follows from the restriction of equation (10) to $D$ that:

$$
\int_{D}\left(n_{1}^{2}-n_{2}^{2}\right)^{2} u_{1}^{2} d x=0
$$

Consequently, we deduce from equations (13)-(14) that:

$$
u_{1}=0 \quad \text { a.e. in } D \text {. }
$$

Using the same argument as in Case 1 , we deduce that $u_{1}=0$ a.e. in $D^{c}=$ $\mathbb{R}^{2} \backslash \bar{D}$ and therefore $u_{1}=0$ a.e. in $\mathbb{R}^{2}$, which contradicts $u_{1}$ being an eigenfunction (i.e., $\left\|u_{1}\right\|_{2} \neq 0$ ).

Remark. The above proof suggests that the conclusion of Proposition 3.1 is still valid when the considered fundamental mode $\left(\beta_{1}, u_{1}\right)$ is replaced by any other pair of guided modes. The multiplicity of the propagation constant does not matter in the proof of the proposition.

The next result states that the previous uniqueness result is no longer valid if the propagation constant value is given without the knowledge of the order of the corresponding guided mode.

Proposition 3.2. The refractive index $n$, solution of ISPP, cannot be uniquely determined in $\mathcal{N}_{1}$ if the propagation constant $\beta$ is given without its associated eigenfield $u$.

Proof. In this situation, it is possible to prove the existence of a sequence of refractive indices $\left(n_{l}\right)_{l} \in \mathcal{N}_{1}$, for which the considered $\beta$ is the propagation constant of the corresponding $l^{\text {th }}$ mode. Indeed, observe that the mapping $F_{1}: n \longmapsto \beta_{1}(n)$ is an increasing function in the following sense: 
for $n, n^{\prime} \in \mathcal{N}_{1}$, such that $n \geq n^{\prime}$, then $F_{1}(n)=\beta_{1}(n) \geq \beta_{1}\left(n^{\prime}\right)=F_{1}\left(n^{\prime}\right)$ [59]. In addition, $F_{1}$ is locally lipschitzian, and therefore continuous from $\mathcal{N}_{1}$ to $\mathbb{R}_{+}^{\star}$ [59]. Consequently, $F_{1}$ defines a bijection from $\mathcal{N}_{1}$ to $\mathbb{R}_{+}^{\star}$. This implies the existence of a unique refractive index $n_{1} \in \mathcal{N}_{1}$, solution of ISPP, such that $\beta$ is the corresponding fundamental mode propagation constant.

Similarly, since the mapping $F_{l}, l \geq 2$, is also a bijection from $\mathcal{N}_{1}$ into $\mathbb{R}_{+}^{\star}$ [59], then we also prove the existence of a sequence of refractive indices $\left(n_{l}\right)_{l \geq 2}$ in $\mathcal{N}_{1}$ such that the considered $\beta$ is also associated to their respective $l^{\text {th }}$ guided mode.

We conclude this section by providing a characterization of the Fréchet derivative of the propagation constants with respect to the refractive index $n$. This result is relevant to the implementation of any Newton-type method for solving ISPP. Indeed, it is well known that the accuracy and the efficiency of Newton-type methods strongly depend on the accuracy level in the computation of the jacobian matrix that occurs at each iteration.

Theorem 3.3. For a given wavenumber $k$, the Fréchet derivative of the mapping $F_{l}(1 \leq l \leq S)$, given by equation (7), with respect to the refractive index profile $n$ in the direction $h \in L^{\infty}\left(\mathbb{R}^{2} ; \mathbb{R}_{+}^{*}\right)$, when it exists, satisfies:

$$
\frac{\partial F_{l}}{\partial n}(n) h=\frac{k^{2} \int_{\mathbb{R}^{2}} n h u_{l}^{2} d x}{\beta_{l} \int_{\mathbb{R}^{2}} u_{l}^{2} d x} .
$$

Proof. Since the guided mode pair $\left(\beta_{l}, u_{l}\right)$ satisfies (5), then we apply the chain rule and obtain:

$$
\Delta\left(\frac{\partial u_{l}}{\partial n} h\right)+\left(k^{2} n^{2}-\beta_{l}^{2}\right) \frac{\partial u_{l}}{\partial n} h+2\left(k^{2} n h-\beta_{l} \frac{\partial \beta_{l}}{\partial n} h\right) u_{l}=0
$$

Next, we multiply equation (17) by $u_{l}$ and integrate over $\mathbb{R}^{2}$. We then obtain:

$$
\int_{\mathbb{R}^{2}}\left[\Delta\left(\frac{\partial u_{l}}{\partial n} h\right)+\left(k^{2} n^{2}-\beta_{l}^{2}\right) \frac{\partial u_{l}}{\partial n} h\right] u_{l} d x+2 \int_{\mathbb{R}^{2}}\left(k^{2} n h-\beta_{l} \frac{\partial \beta_{l}}{\partial n} h\right) u_{l}^{2}=0
$$

Furthermore, we integrate equation (18) by parts and obtain:

$$
\int_{\mathbb{R}^{2}}\left[\Delta u_{l}+\left(k^{2} n^{2}-\beta_{l}^{2}\right) u_{l}\right] \frac{\partial u_{l}}{\partial n} h d x+2 \int_{\mathbb{R}^{2}}\left(k^{2} n h-\beta_{l} \frac{\partial \beta_{l}}{\partial n} h\right) u_{l}^{2}=0
$$

Note that, similarly to the derivation of the variational formulations in [63][64], equation (19) is obtained from (18) in two steps. We first perform 
the integration in the distribution sense. We then use the standard density argument of $\mathcal{D}\left(\mathbb{R}^{2}\right)$ into $H^{2}\left(\mathbb{R}^{2}\right)$.

Finally, it follows from equations (5) and (19) that

$$
\int_{\mathbb{R}^{2}}\left(k^{2} n h-\beta_{l} \frac{\partial \beta_{l}}{\partial n} h\right) u_{l}^{2}=0
$$

which concludes the proof of Theorem 3.3.

\section{Solution Methodology}

We propose a Tikhonov-regularized Newton procedure for solving ISPP [65][66], since regularized iterative methods appear to be the primary candidates for solving nonlinear and ill-posed problems (see, e.g., [67], and the references therein). The Newton algorithm addresses the nonlinear aspect of ISPP, whereas the Tikhonov regularization procedure is incorporated to address its ill-posed nature [68]-[69].

\subsection{Parametrization}

We assume that the sought-after refractive index profile $n$ is in $\mathcal{N}_{1}$ and can be approximated by a set of trial solutions given by the following parametrization:

$$
n(\mathbf{x}) \approx \sum_{m=1}^{N_{P}} \alpha_{m} g_{m}(|\mathbf{x}|) \quad ; \quad \forall \mathbf{x} \in \mathbb{R}^{2}
$$

where:

- the parameter $N_{P}$ is a positive integer representing the number of parameters. $N_{P}$ is typically between 2 and 4 , for most refractive index profiles of practical interest [60], [56]-[58]. Note that the numerical results reported in [54] were obtained with $N_{P}=2$ and 3 only.

- the parameters $\alpha_{1}, \alpha_{2}, \cdots, \alpha_{N_{P}}$ are real numbers. They represent the unknown coefficients to be determined.

- $\left(g_{m}\right)_{1 \leq m \leq N_{P}}$ is a selected set of real-valued polynomial functions such that:

$i$. For $1 \leq m \leq N_{P}-1, g_{m}$ is a piecewise polynomial function of degree $(m-1)$, whose support is contained in the core $\Omega$ of the fiber. 
ii. $g_{N_{P}}$ is the characteristic function of the fiber cladding, i.e., $g_{N_{P}}(\mathbf{x})=$ 0 if $\mathbf{x} \in \Omega$ and $g_{N_{P}}(\mathbf{x})=1$ elsewhere.

Basis functions for parametrizing respectively a refractive step-index profile and a refractive graded-index profile of a circular-shaped optical fiber are depicted in Figures 2 and 3, for illustrative purposes. Observe that it is possible to employ other basis functions such as trigonometric or B-spline functions that are often encountered when solving inverse problems (see, e.g., [67]). This type of bases is more appropriate for refractive index profiles that are not function of the radial direction $r$, but depend on $\mathbf{x}=\left(x_{1}, x_{2}\right)$, particularly for optical fibers with arbitrary cross-sectional boundaries. We have adopted here these polynomial-type functions for mainly two reasons:

(a) The class of refractive index profiles that we consider (see (3)-(4)) is always piecewise continuous in $\Omega$ and constant in $\Omega^{c}$. Hence, these functions appear to form an appropriate basis for approximating this class of profiles.

(b) Since the proposed solution methodology employs a finite element method for solving the direct eigenvalue problem (see Section 4.3), the use of polynomial functions in the parametrization can be easily "blended" in the finite element approximation without significantly increasing the computational complexity of the method.

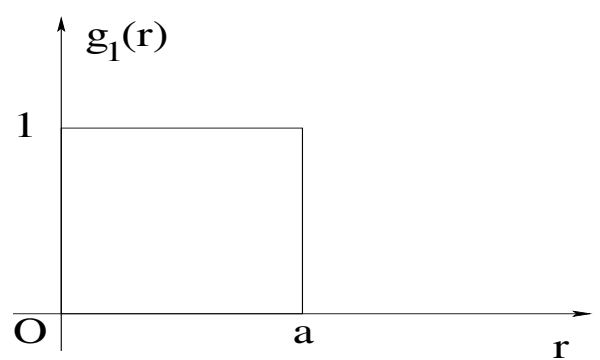

(a)

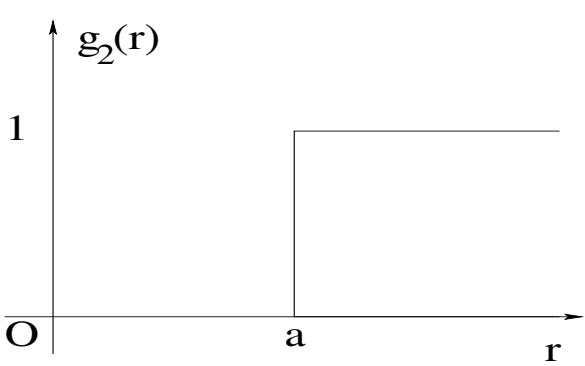

(b)

Figure 2: Basis functions for parametrizing refractive step-index profiles in the case of a circular-shaped optical fiber. $N_{P}=2$.

\subsection{Newton iteration equation}

Assume $n^{(j)}=\sum_{m=1}^{N_{P}} \alpha_{m}^{(j)} g_{m}$ to be the computed refractive index profile at iteration $j$ that approximates the solution $n^{\star}$ of ISPP. Then, equation (8) is 


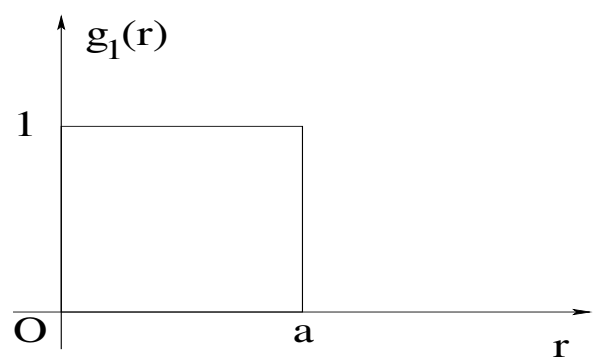

(a)

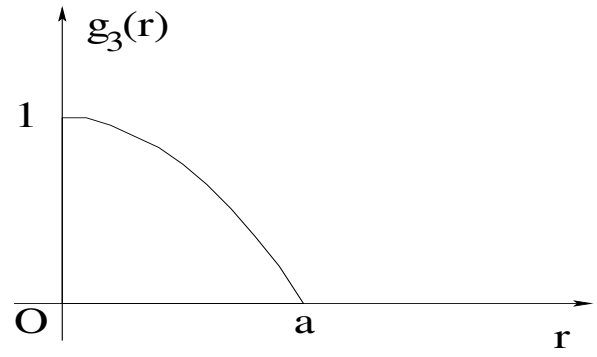

(c)

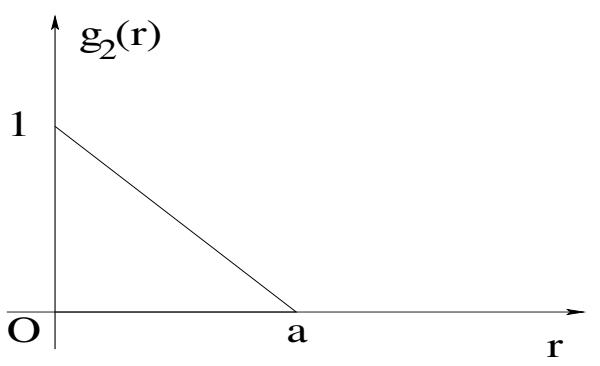

(b)

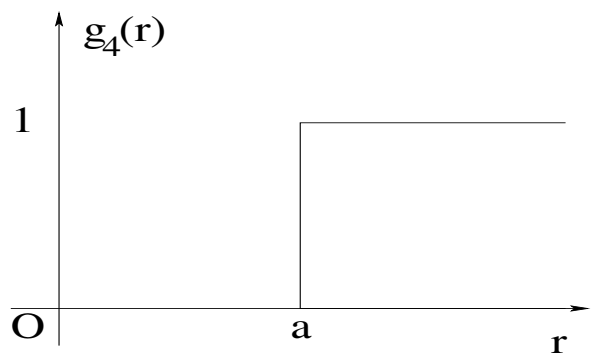

(d)

Figure 3: Basis functions for parametrizing refractive graded-index profiles in the case of a circular-shaped optical fiber. $N_{P}=4$.

replaced by the Newton iteration equation:

$$
\mathbf{J}_{\mathbf{F}}^{(j)} \delta n^{(j)}=\boldsymbol{\beta}_{I}-\mathbf{F}_{I}\left(n^{(j)}\right)
$$

where $\delta n^{(j)}$ is the update vector given by:

$$
\delta n^{(j)}=\sum_{m=1}^{N_{P}} \delta \alpha_{m}^{(j)} g_{m}
$$

and the updated index profile $n^{(j+1)}$ is given by:

$$
n^{(j+1)}=n^{(j)}+\delta n^{(j)},
$$

where $\mathbf{J}_{\mathbf{F}}^{(j)}$ is the jacobian matrix of the operator $\mathbf{F}_{I}=\left(F_{1}, \cdots, F_{I}\right)^{T}$, evaluated at $n^{(j)}$, i.e.

$$
\mathbf{J}_{\mathbf{F}}^{(j)}=\left(\frac{\partial \beta_{l}^{(j)}}{\partial n}\left(n^{(j)}\right) g_{m}\right) \begin{aligned}
& \begin{array}{l}
1 \leq l \leq I \\
1 \leq m \leq N_{P}
\end{array}
\end{aligned} .
$$

$\beta_{l}^{(j)}=F_{l}\left(n^{(j)}\right) \quad(1 \leq l \leq I)$ designates the propagation constant of the $l^{\text {th }}$ guided mode, solution of EVP, for the refractive index profile $n^{(j)}$, and 
$\left(g_{m}\right)_{1 \leq m \leq N_{P}}$ is the considered basis of functions. The entries of the jacobian matrix $\mathbf{J}_{\mathbf{F}}^{(j)}$ are given, as stated in Theorem 3.3, by equation (16) in which $h$ is replaced by the basis functions $g_{m}$, introduced in Paragraph 4.1 (see Figures 2-3).

Note that, at the algebraic level, if the number of parameters $N_{P}$ is equal to the number of the measurements $I$, then equation (22) is a square linear system. However, if $N_{P}$ is larger than $I$, the linear system (22) is rectangular. In this case, we solve this system in the least-squares sense, that is, equation (22) is replaced by:

$$
\mathbf{J}_{\mathbf{F}}^{T^{(j)}} \mathbf{J}_{\mathbf{F}}^{(j)} \delta n^{(j)}=\mathbf{J}_{\mathbf{F}}^{T^{(j)}}\left(\boldsymbol{\beta}_{I}-\mathbf{F}_{I}\left(n^{(j)}\right)\right),
$$

where $T$ stands for the transpose of a matrix. Last, since ISPP is an ill-posed problem, we employ the standard Tikhonov regularized procedure to restore the stability [68]-[69]. Therefore, we replace equation (22) or equation (26) by the following compact regularized Newton-type equation:

$$
\left(\mathbf{E}^{(j)} \mathbf{J}_{\mathbf{F}}^{(j)}+\mu \mathbf{I}_{\mathbf{N}_{\mathbf{P}}}\right) \delta n^{(j)}=\mathbf{E}^{(j)}\left(\boldsymbol{\beta}_{I}-\mathbf{F}_{I}\left(n^{(j)}\right)\right)
$$

where $\mathbf{E}^{(j)}$ is a $N_{P} \times I$ matrix given by:

$$
\mathbf{E}^{(j)}=\left\{\begin{array}{lll}
\mathbf{I}_{\mathbf{N}_{\mathbf{P}}} & \text { if } & N_{P}=I \\
\mathbf{J}_{\mathbf{F}}^{T^{(j)}} & \text { if } & N_{P}>I,
\end{array}\right.
$$

and $\mu$ is a positive number called the regularized parameter. There are various strategies for selecting the "optimal" value of $\mu$ (see, e. g. [70]-[73]). Due to the small size of the resulting linear system, we propose a trial and error strategy for finding the optimal value of $\mu$. This consists in sweeping $\mu$ over a large interval of positive real numbers and evaluating the residual for each value of $\mu$, and we then select the value of $\mu$ that leads to the minimum residual (up to the noise level).

\subsection{Efficient solver for the direct eigenvalue problem EVP}

The proposed regularized Newton algorithm calls for the solution of the forward eigenvalue problem EVP. To this end, we employ the direct solver developed in references [74]-[75]. This solver requires to first reformulating EVP 
in a bounded domain (see Figure 4(a)) as follows:

$$
(\mathcal{E V} \mathcal{P})\left\{\begin{array}{lr}
\text { Find } \beta \in] k n_{\infty}, k n_{+}\left[\text {and } u \in L^{2}\left(\Omega_{\Sigma}\right) ; u \neq 0\right. \text { such that: } \\
\Delta u+k^{2} n^{2} u=\beta^{2} u & \text { in } \Omega_{\Sigma} \\
{[u]=\left[\frac{\partial u}{\partial \nu}\right]=0} & \text { on } \Gamma=\partial \Omega \\
\frac{\partial u}{\partial \nu}+\left(\sqrt{\beta^{2}-k^{2} n_{\infty}^{2}}+\frac{\mathcal{K}}{2}\right) u=0 & \text { on } \Sigma=\partial \Omega_{\Sigma},
\end{array}\right.
$$

where $\mathcal{K}$ denotes the curvature of the employed artificial boundary $\Sigma$. In the particular case of $\Sigma$ being a circular-shaped boundary of radius $R, \mathcal{K}=1 / R$. Then, we apply a linear finite element approximation [76]-[77] to calculate the solutions of the truncated eigenvalue problem $\mathcal{E} \mathcal{V P}$. This leads to the solution of the following quadratic eigenvalue problem:

$$
A x+\lambda B x+\sqrt{\lambda} C x=0
$$

where:

- $\lambda=\beta^{2}-k^{2} n_{\infty}^{2}$ is the eigenvalue.

- $x$ is the corresponding eigenvector. $x \in \mathbb{R}^{N}$ with $N$ being the number of degrees of freedom of the FEM approximation.

- $A, B$, and $C$ are symmetric matrices. $A$ is the sum of a stiffness matrix, a mass-like matrice, and a mass-like matrix on $\Sigma$ that results from the term containing the curvature $\mathcal{K}$ in equation (31). $B$ is a mass matrix and therefore $B$ is positive definite. $C$ is a mass-type matrix defined on $\Sigma$. $C$ is a quasi-tridiagonal matrix.

To solve numerically the quadratic eigenvalue problem given by (32), we transform it into a generalized eigenvalue problem of the form [74]-[75]:

$$
\widetilde{A} z=\sqrt{\lambda} \widetilde{B} z
$$

where

$$
\widetilde{A}=\left[\begin{array}{cc}
-C & -B \\
A & 0
\end{array}\right]
$$

and

$$
\widetilde{B}=\left[\begin{array}{cc}
B & 0 \\
0 & B
\end{array}\right]
$$

We compute the pairs of eigensolutions $(\sqrt{\lambda}, z)$ by employing the Implicitly Restarted Arnoldi Method IRAM [78], which is an iterative algorithm of QRtype [79]-[80]. 


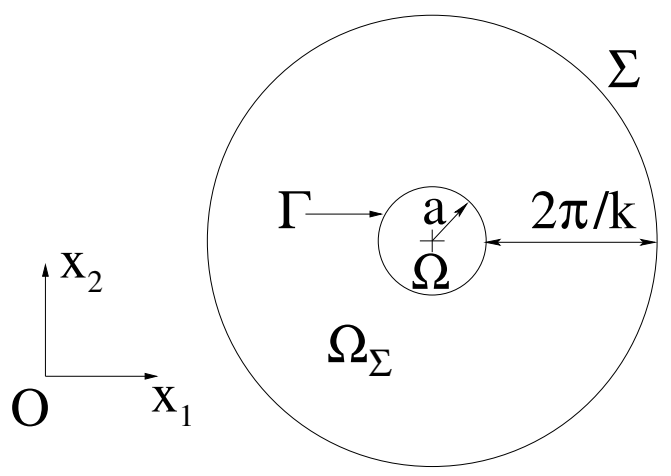

(a)

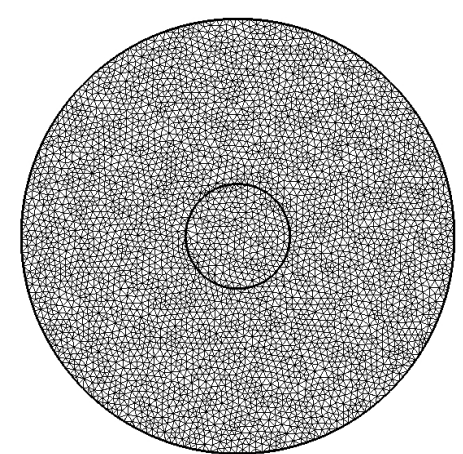

(b)

Figure 4: (a) The computational domain for a circular-shaped optical fiber and (b) an illustrative finite element mesh using triangular-shaped elements.

\subsection{Algorithm summary}

The proposed algorithm can be summarized as follows:

Step 0. Initialization. The proposed algorithm requires the following initial data:

- A prescribed shape of the core-cladding interface $\Gamma=\partial \Omega$ of the considered optical fiber.

- A set of $N_{F}$ wavenumbers $k_{1}, \cdots, k_{N_{F}}$ defining the frequency regime of the guided wave propagation.

- A set of the desired/measured guided modes that propagate in the considered fiber for the respective prescribed wavenumbers $k_{l}, 1 \leq l \leq$ $N_{F}$. For each $k_{l}$, its corresponding desired/measured guided modes are characterized by a set of pairs consisting of the propagation constants and their corresponding guided fields, i.e., for each wavenumber $k_{l}$, we have the set of desired/measured pairs:

$$
\left(\widetilde{\beta_{1_{l}}^{\star}}, \widetilde{u_{1_{l}}^{\star}}\right),\left(\widetilde{\beta_{2_{l}}^{\star}}, \widetilde{u_{2_{l}}^{\star}}\right), \cdots,\left(\widetilde{\beta_{I_{l}}^{\star}}, \widetilde{u_{I_{l}}^{\star}}\right), \quad 1 \leq l \leq N_{F} .
$$

Note that these modes are listed with their multiplicities, i.e.

$$
\widetilde{\beta_{1_{l}}^{\star}}<\widetilde{\beta_{2_{l}}^{\star}} \leq \widetilde{\beta_{3_{l}}^{\star}} \leq \cdots \leq \widetilde{\beta_{I_{l}}^{\star}}, \quad 1 \leq l \leq N_{F} .
$$

The tilde indicates that the data (when measured) are possibly tainted with errors. 
- An initial parameter vector $\left(n_{1}^{(0)}, \cdots, n_{N_{P}}^{(0)}\right)$ representing the initial refractive index profile:

$$
n^{(0)}=\sum_{m=1}^{N_{P}} n_{m}^{(0)} g_{m}
$$

The admissible values of these parameters are arbitrarily selected, i.e., they are "blind" guessed values.

Step 1. Apply Newton Iteration. This requires the accomplishment of the following three tasks, at the algorithm $j^{\text {th }}$ iteration $(j=0,1,2, \cdots)$ :

$i$. Solve $\mathcal{E} \mathcal{V P}$ with the refractive index $n^{(j)}$ and for each wavenumber $k_{l}, 1 \leq l \leq N_{F}$, to obtain a set of eigenpairs

$$
\left(\left(\beta_{1_{l}}^{(j)}, u_{1_{l}}^{(j)}\right), \cdots,\left(\beta_{I_{l}}^{(j)}, u_{I_{l}}^{(j)}\right)\right)_{1 \leq l \leq N_{F}} .
$$

ii. Evaluate the jacobian entries given by equation (25) using equation (16), the refractive index $n^{(j)}$, and the computed eigenpairs:

$$
\left(\left(\beta_{1_{l}}^{(j)}, u_{1_{l}}^{(j)}\right), \cdots,\left(\beta_{I_{l}}^{(j)}, u_{I_{l}}^{(j)}\right)\right)_{1 \leq l \leq N_{F}}
$$

iii. Solve the regularized Newton iteration equation given by (27) to evaluate $\delta n^{(j)}$ :

$$
\left(\mathbf{E}^{(j)} \mathbf{J}_{\mathbf{F}}^{(j)}+\mu \mathbf{I}_{\mathbf{N}_{\mathbf{P}}}\right) \delta n^{(j)}=\mathbf{E}^{(j)}\left(\widetilde{\boldsymbol{\beta}_{I}^{\star}}-\mathbf{F}_{I}\left(n^{(j)}\right)\right)
$$

where $\mathbf{E}^{(j)}$ is given by (28).

Step 2. Stopping criteria. We monitor the convergence of the algorithm at iteration $j(j=0,1,2, \cdots)$ by evaluating both the relative residual on the propagation constants:

$$
\operatorname{Error}^{(j)}=\frac{\left(\sum_{l=1}^{N_{F}}\left|\widetilde{\beta_{1_{l}}^{\star}}-\beta_{1_{l}}^{(j)}\right|^{2}+\cdots+\left|\widetilde{\beta_{I_{l}}^{\star}}-\beta_{I_{l}}^{(j)}\right|^{2}\right)^{1 / 2}}{\left(\sum_{l=1}^{N_{F}}\left|\widetilde{\beta_{1_{l}}^{\star}}\right|^{2}+\cdots+\left|\widetilde{\beta_{I_{l}}^{\star}}\right|^{2}\right)^{1 / 2}}
$$

and the magnitude of the refractive index profile update:

$$
\max _{1 \leq m \leq N_{p}}\left|\delta n_{m}^{(j)}\right|
$$


We stop the algorithm at iteration $j$ when one of these two values attains a prescribed tolerance level $\varepsilon$. Note that we also evaluate, at each iteration $j(j=0,1,2, \cdots)$, the relative residual on the fields, i.e:

$$
\text { Error2 }^{(j)}=\frac{\left(\sum_{l=1}^{N_{F}}\left\|\widetilde{u_{1_{l}}^{\star}}-u_{1_{l}}^{(j)}\right\|_{2}^{2}+\cdots+\left\|\widetilde{u_{I_{l}}^{\star}}-u_{I_{l}}^{(j)}\right\|_{2}^{2}\right)^{1 / 2}}{\left(\sum_{l=1}^{N_{F}}\left\|\widetilde{u_{1_{l}}^{\star}}\right\|_{2}^{2}+\cdots+\left\|\widetilde{u_{I_{l}}^{\star}}\right\|_{2}^{2}\right)^{1 / 2}}
$$

This quantity is however used as a "discrimination" tool, i.e., to identify the order of the computed modes as well as their corresponding polarization [56][58].

\subsection{Computational complexity}

The proposed solution methodology summarized in Paragraph 4.4 requires, at each Newton iteration, the following:

- The computation, for each considered wavenumber $k_{l}\left(1 \leq l \leq N_{F}\right)$, of the first $I_{l}$ eigenpairs $\left(\beta_{1_{l}}, u_{1_{l}}\right), \cdots,\left(\beta_{I_{l}}, u_{I_{l}}\right)$. This is performed by applying IRAM algorithm (which is a QR-type method) [78] to a generalized eigenvalue system whose size is $2 N \times 2 N$, where $N$ is the number of degrees of freedom of the FEM approximation. Note that the application of IRAM calls for solving non-symmetric linear systems, which is accomplished using GMRES procedure [79].

- The computation of the jacobian entries, for each considered wavenumber $k_{l}\left(1 \leq l \leq N_{F}\right)$, by evaluating $N_{P} \times I_{l}$ integrals. This can be performed using a Gauss-type quadrature [81]-[82]. However, since we have employed an "overkill" mesh for solving the direct eigenvalue problem EVP, the size of the triangles is very small compared to the variations of the integrands. Hence, it appears reasonable to assume the integrand over each FEM triangle to be constant and equal to the average of its values on the nodes of the considered triangle. The obtained results indicate that this simple procedure is very accurate and cost-effective.

- The determination of the profile update $\delta n$ by solving a small $N_{P} \times N_{P}$ linear system that can be executed analytically by simply applying a direct method [83]. However, in this work, we inverted this system 
analytically when the order of parameter $N_{P}$ is either 2 or 3 (see Sections 5.1 and 5.2). Consequently, we have observed that $\mu=0$ is the optimal value of the regularization parameter, i.e., there is no need to regularize since the inversion is analytically performed and therefore is "exact" when the number of the sought-after parameters is less or equal to 3. On the other hand, when $N_{P}=5$ or 6 (see Section 5.3), the algorithm does not converge without regularization.

\section{$5 \quad$ Illustrative Numerical Results}

We present numerical results to illustrate the potential of the proposed Newton algorithm for efficiently determining the refractive index profile from the knowledge of some guided modes. These results were obtained in the case of an optical fiber whose core-cladding interface $\Gamma$ is a circular-shaped boundary (see Figure 4(a)). In all of the numerical experiments, we set the radius $a$ of $\Gamma$ to be $a=0.4 \mu \mathrm{m}$ and we use only one frequency whose wavenumber is $k=5 \times 10^{6} \mathrm{~m}^{-1}$. Note that we have fixed the values of these two optogeometric parameters so that we can use the same finite element resolution in all experiments, i.e., there is no need to generate a new mesh. On the other hand, the refractive index profile parameters $n_{+}$and $n_{\infty}$ will have their values changed to ensure that the value of the normalized frequency $V=k a \sqrt{n_{+}^{2}-n_{\infty}^{2}}$ is sufficiently large to allow the needed number of guided modes to propagate in the fiber [84]-[85]. Furthermore, we use the following discretization parameters:

- The exterior artificial boundary $\Sigma$ is circular-shaped of radius $R$ and is located at one wavelength from the core-cladding interface $\Gamma$ (see Figure 4(a)). This distance is considered to be far enough to prevent any reflections, as demonstrated in references [74]-[75].

- We use a linear finite element approximation with 50 elements per wavelength. This discretization appears to be fine enough to ensure the computation of the guided modes with a high accuracy level [74][75].

In what follows, we present results for determining three major classes of refractive index profiles that are of practical interest: step-index, gradedindex, and W-refractive index profiles [56]-[58], [60], [84]. 


\subsection{Retrieving refractive step-index profiles}

The goal of this section is to determine the parameters corresponding to a refractive step-index profile. To this end, we consider the class of refractive index profiles of the form:

$$
n(x)=\left\{\begin{array}{lll}
n_{+} & ; \quad x \in \Omega \\
n_{\infty} & ; \quad x \in \Omega^{e} .
\end{array}\right.
$$

We present the results of two numerical experiments. In the first one, the goal is to determine the refractive index of the core of the fiber only, whereas in the second one, we recover both indices. We must point out that the measured guided modes are synthetic data obtained by solving the following dispersion equation (see equation (A9), page 1574, in reference [74]):

$$
\lambda_{1} \frac{\mathbf{J}_{\nu+1}\left(\lambda_{1}\right)}{\mathbf{J}_{\nu}\left(\lambda_{1}\right)}=\lambda_{2} \frac{\mathbf{K}_{\nu+1}\left(\lambda_{2}\right)}{\mathbf{K}_{\nu}\left(\lambda_{2}\right)} ; \nu=0,1,2, \cdots
$$

where $\lambda_{1}=a \sqrt{k^{2} n_{+}^{2}-\beta^{2}}$ and $\lambda_{2}=a \sqrt{\beta^{2}-k^{2} n_{\infty}^{2}} . \mathbf{J}_{\nu}\left(\operatorname{resp} . \mathbf{K}_{\nu}\right)$ is the Bessel function (resp. the modified Bessel function) of the first kind [81].

\subsubsection{Experiment 1: Partial parameters recovery}

The goal of this experiment is to retrieve one refractive index parameter from the knowledge of one pair of guided modes. We choose the unknown refractive index profile parameter to be $n_{+}$, the refractive index of the core of the fiber, and we assume that:

$$
n_{+}-n_{\infty}=0.01
$$

This means that we consider here a one-parameter inverse problem. The target refractive index profile value is $n_{+}^{\star}=50.005$ whereas the initial value is $n_{+}^{(0)}=200.005$. The measured guided mode corresponding to the target profile $n_{+}^{\star}$ is the fundamental mode $L P_{01}[56]-[58]$ whose propagation constant, computed from equation (39), is:

$$
\beta_{1}^{\star}=249.996
$$

The initial propagation constant value corresponding to the initial refractive index profile $n^{(0)}$ is:

$$
\beta_{1}^{(0)}=1000.014 \text {. }
$$

We also assess the sensitivity of the performance efficiency of the proposed algorithm to the noise level in the data. To this end, the propagation constant $\beta_{1}^{\star}$ was respectively tainted with three different levels of white noise: 
$5 \%, 10 \%$ and $20 \%$. Note that the noisy values are denoted by $\widetilde{\beta_{1}^{\star}}$. The results are reported in Table 1 and Figures 5-6. The following observations are noteworthy:

- The initial value of the refractive index parameter is selected outside the pre-asymptotic convergence region. Indeed, the relative error on the refractive index profile is about $300 \%$. In addition, equations (41)(42) show that the use of this initial guess leads to the computation of a propagation constant with relative residuals ranging from $200 \%$ to $300 \%$, depending on the noise level. Furthermore, as indicated in Figures $6(\mathrm{a})$ and $6(\mathrm{~b})$, the isovalues of the exact field vary between 0.0002 and 0.037 , whereas the ones corresponding to the initial guess range between $1.06 \times 10^{-7}$ and 0.05 . Clearly, Experiment 1 is performed with a "blind" initial guess value $n_{+}^{\star}$.

- Figure 5 illustrates the fast convergence and the robustness to the noise effect of the proposed solution methodology. More specifically, one can observe that the relative residual drops from the initial value of over $200 \%$ to about the noise level in -at most- 3 iterations, for all considered noise levels. In addition, Figure 5 reveals that the convergence is monotone with almost no oscillations. This unusual behavior when solving inverse-type problems is most likely due to the fact that the algorithm is applied to a one-parameter inverse problem and therefore the instability effects, if any, seem to be barely noticeable.

- At convergence, the refractive index parameter $n_{+}$is delivered with an accuracy up to the noise level (see Table 1). On the other hand, Table 1 indicates that the proposed algorithm delivers the guided field $u_{1}$ with an excellent accuracy level even when the noise level is $20 \%$ (see Figure 6). 


\begin{tabular}{|c||c|c|c|c|}
\hline $\begin{array}{c}\text { Initial } \\
\text { noise level } \\
\text { on } \beta_{1}^{\star}(\%)\end{array}$ & $\begin{array}{c}\text { Relative } \\
\text { residual (\%) }\end{array}$ & $\begin{array}{c}\text { Relative } \\
\text { error } \\
\text { on } n^{\star}(\%)\end{array}$ & $\begin{array}{c}\text { Relative } \\
\text { error } \\
\text { on } \beta_{1}^{\star}(\%)\end{array}$ & $\begin{array}{c}\text { Relative } \\
\text { error } \\
\text { on } u_{1}^{\star}(\%)\end{array}$ \\
\hline \hline 0 & 0.852 & 0.852 & 0.852 & 0.308 \\
\hline 5 & 3.042 & 8.193 & 8.194 & 3.063 \\
\hline 10 & 2.734 & 13.005 & 13.007 & 4.701 \\
\hline 20 & 2.229 & 22.671 & 22.675 & 7.667 \\
\hline
\end{tabular}

Table 1: Sensitivity of the relative residual and the relative error, at convergence, to the noise level for Experiment 1.

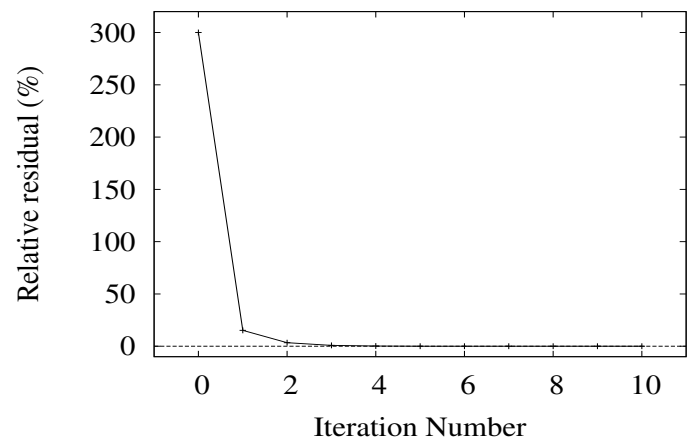

(a) Noise free

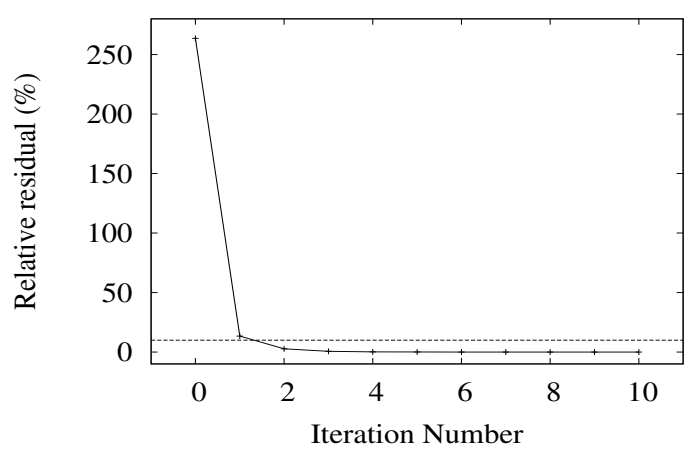

(c) Noise level $10 \%$.

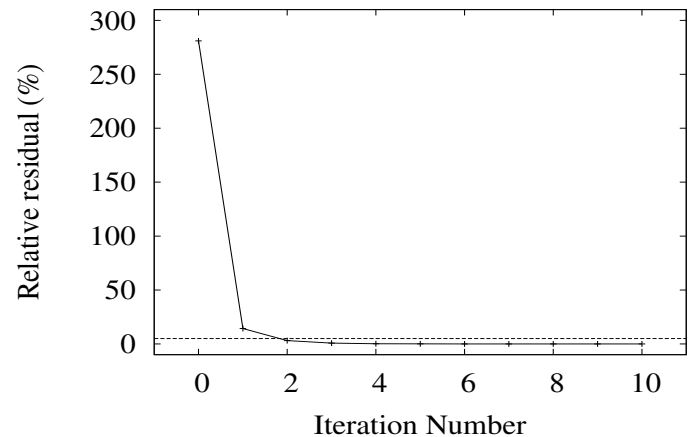

(b) Noise level $5 \%$.

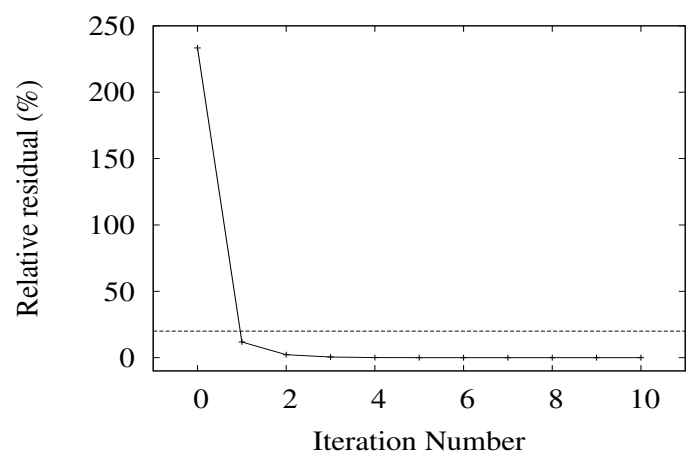

(d) Noise level $20 \%$.

Figure 5: Convergence history. Sensitivity of the relative residual given by equation (35) to the noise level on the propagation constant $\widetilde{\beta_{1}^{\star}}$. 


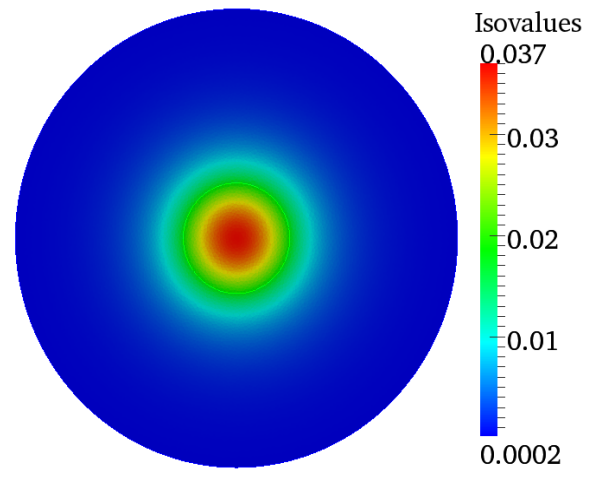

(a) Analytical

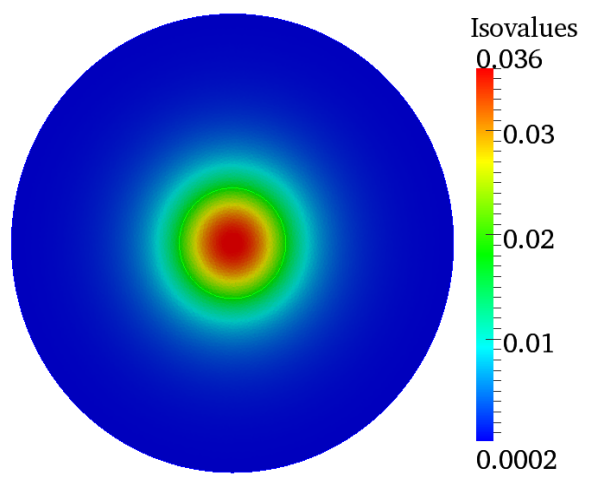

(c) Computed with noise free

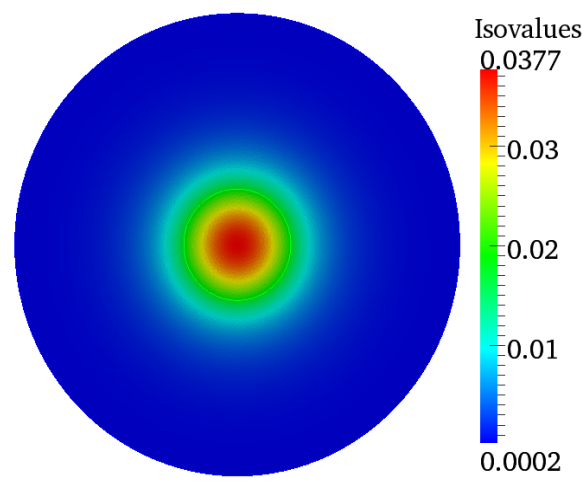

(e) Computed with $10 \%$ noise level

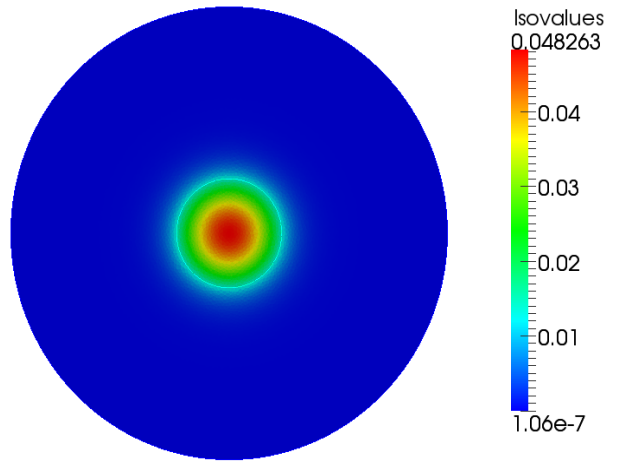

(b) Initial

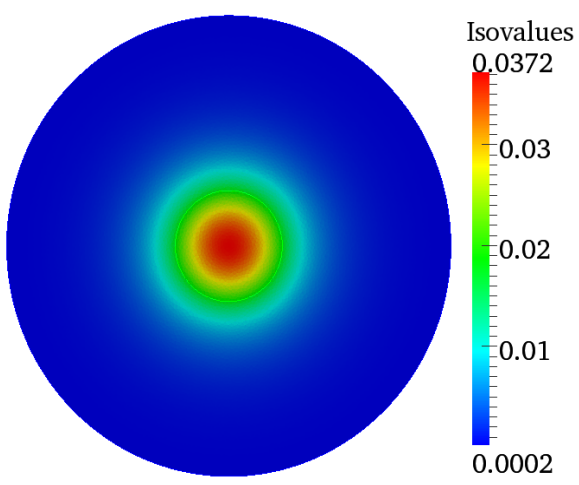

(d) Computed with 5\% noise level

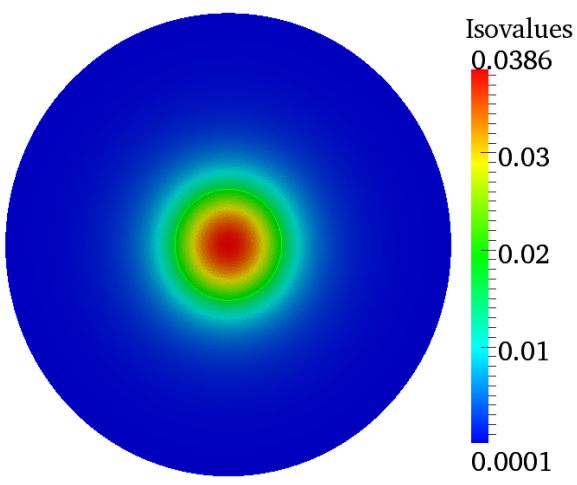

(f) Computed with $20 \%$ noise level

Figure 6: Isovalues corresponding to the fundamental mode $L P_{01}$. Analytic vs. computed fields for various noise levels on $\widetilde{\beta_{1}^{\star}}$. 


\subsubsection{Experiment 2: Full parameters recovery}

The goal here is to retrieve the two refractive index parameters when two measured/desired eigenmodes are given. The unknown refractive index parameters are $n_{+}$and $n_{\infty}$ (see equation (38) and Figure 2 ). The values of the target and initial refractive index profiles, $n^{\star}$ and $n^{(0)}$, are reported in Table 2. The measured guided modes corresponding to the target profile $n^{\star}$ are the fundamental mode $L P_{01}$ and the second mode $L P_{11}$ [56]-[58]. The propagation constants corresponding to these two modes are obtained from equation (39), and their values are:

$$
\boldsymbol{\beta}_{2}^{\star}=\left(\beta_{1}^{\star}, \beta_{2}^{\star}\right)^{T}=(1000.014,999.997)^{T} .
$$

For the initial refractive index profile $n^{(0)}$ (see Table 2), its propagation constants corresponding respectively to modes $L P_{01}$ and $L P_{11}$ [56], are:

$$
\boldsymbol{\beta}_{2}^{(0)}=\left(\beta_{1}{ }^{(0)}, \beta_{2}{ }^{(0)}\right)^{T}=(2000.018,2000.007)^{T} .
$$

Similarly to Experiment 1, we assess the sensitivity of the proposed algorithm to the noise level in the data by respectively adding to $\boldsymbol{\beta}_{2}^{\star}$ three levels of white noise: $5 \%, 10 \%$ and $20 \%$, and thereafter denoted by $\widetilde{\boldsymbol{\beta}_{2}^{\star}}$.

The obtained results are reported in Table 3 and Figures 7-9. The following observations are noteworthy:

\begin{tabular}{|c||c|c|}
\hline $\begin{array}{c}\text { Refractive index } \\
\text { profile parameters }\end{array}$ & $n_{+}$ & $n_{\infty}$ \\
\hline \hline Target & 200.005 & 199.995 \\
\hline Initial & 400.005 & 399.980 \\
\hline
\end{tabular}

Table 2: Target vs. initial refractive index profile parameters for Experiment 2 . 


\begin{tabular}{|c||c|c|c|c|c|}
\hline $\begin{array}{c}\text { Initial } \\
\text { noise level } \\
\text { on } \boldsymbol{\beta}_{2}^{\star}(\%)\end{array}$ & $\begin{array}{c}\text { Relative } \\
\text { residual (\%) }\end{array}$ & $\begin{array}{c}\text { Relative } \\
\text { error } \\
\text { on } n^{\star}(\%)\end{array}$ & $\begin{array}{c}\text { Relative } \\
\text { error } \\
\text { on } \boldsymbol{\beta}_{2}^{\star}(\%)\end{array}$ & $\begin{array}{c}\text { Relative } \\
\text { error } \\
\text { on } u_{1}^{\star}(\%)\end{array}$ & $\begin{array}{c}\text { Relative } \\
\text { error } \\
\text { on } u_{2}^{\star}(\%)\end{array}$ \\
\hline \hline 0 & 0.0 & $4.349 \times 10^{-5}$ & 0.0 & 0.18468 & 1.26672 \\
\hline 5 & $2.926 \times 10^{-5}$ & 4.999 & 5.0 & 5.19346 & 7.83993 \\
\hline 10 & $8.864 \times 10^{-5}$ & 9.999 & 10.0 & 9.21879 & 13.35070 \\
\hline 20 & $6.732 \times 10^{-5}$ & 19.989 & 20.0 & 22.15990 & 28.71202 \\
\hline
\end{tabular}

Table 3: Sensitivity of the relative residual and the relative error, at convergence, to the noise level for Experiment 2.

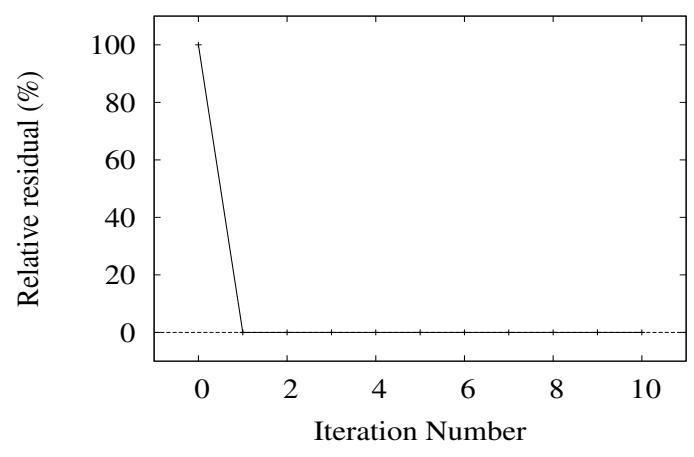

(a) Noise free

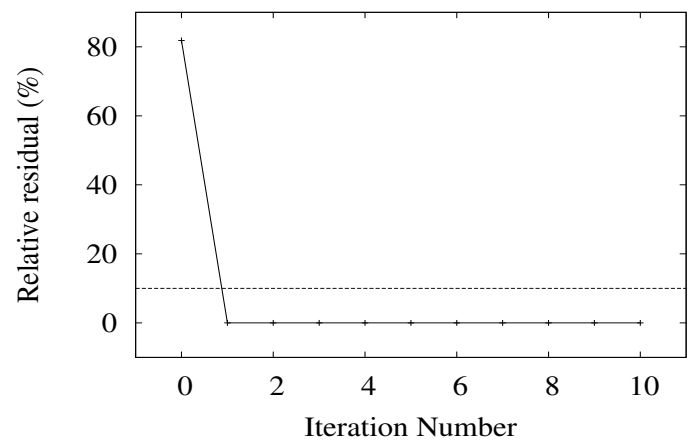

(c) Noise level $10 \%$.

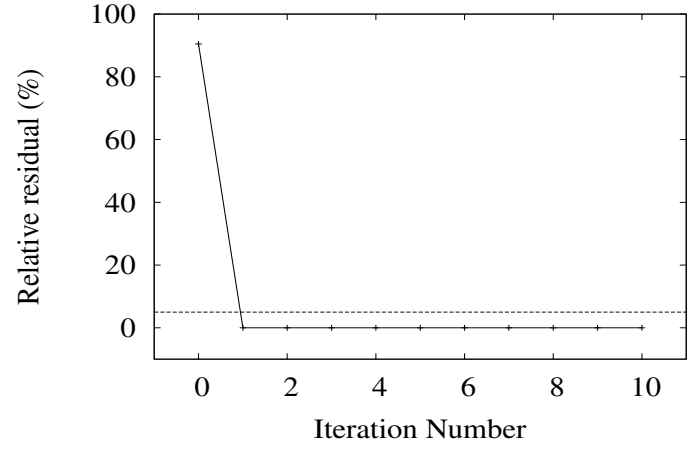

(b) Noise level $5 \%$.

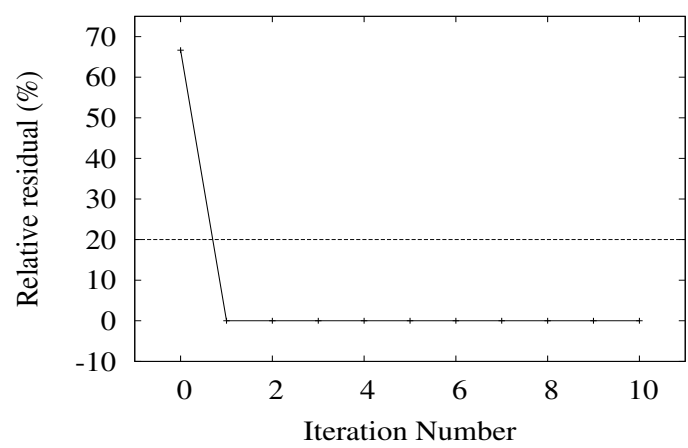

(d) Noise level $20 \%$.

Figure 7: Convergence history. Sensitivity of the relative residual given by equation (35) to the noise level on the propagation constants $\widetilde{\boldsymbol{\beta}_{2}^{\star}}$ for Experiment 2. 


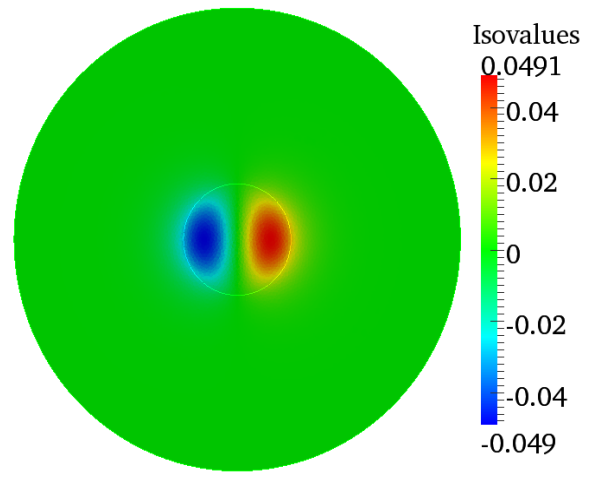

(a) Analytical

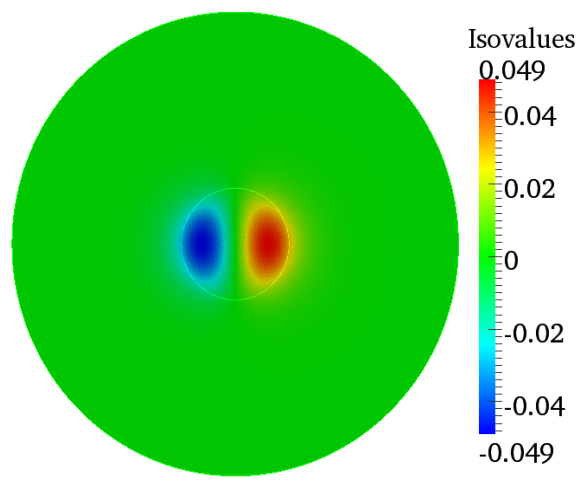

(c) Computed with $0 \%$ noise level

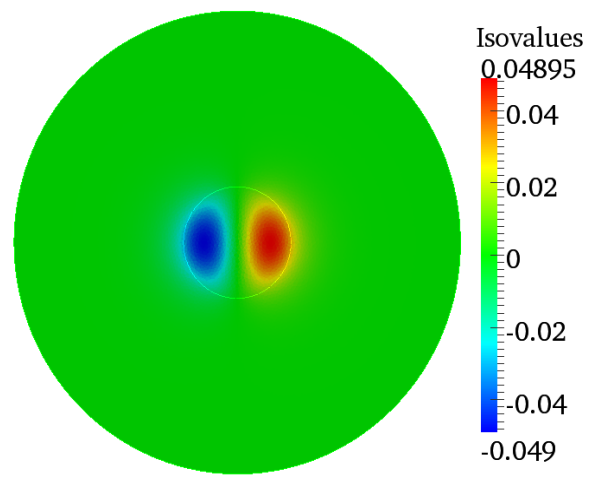

(e) Computed with $10 \%$ noise level

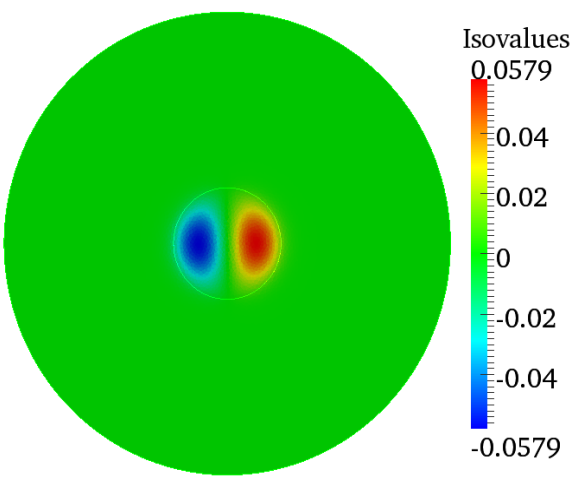

(b) Initial

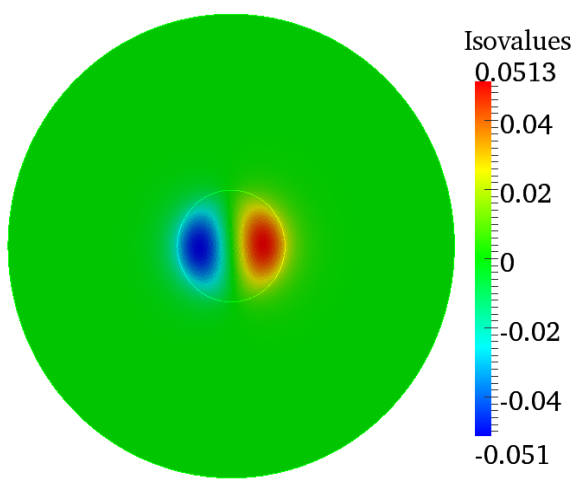

(d) Computed with $5 \%$ noise level

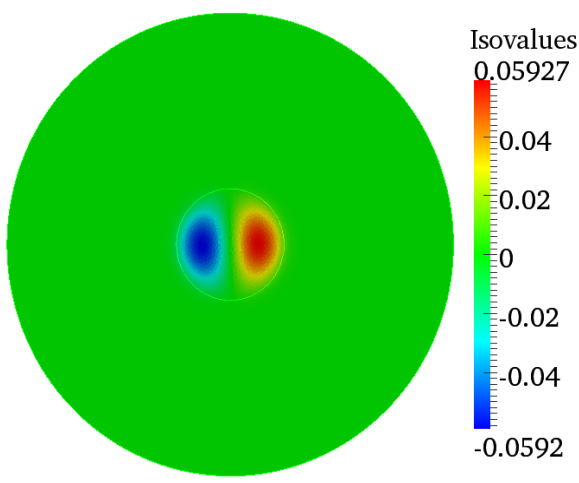

(f) Computed with $20 \%$ noise level

Figure 8: Isovalues corresponding to the guided mode $L P_{11}^{x_{1}}$. Analytic vs. computed fields for various noise levels on $\widetilde{\boldsymbol{\beta}_{2}^{\star}}$ for Experiment 2. 


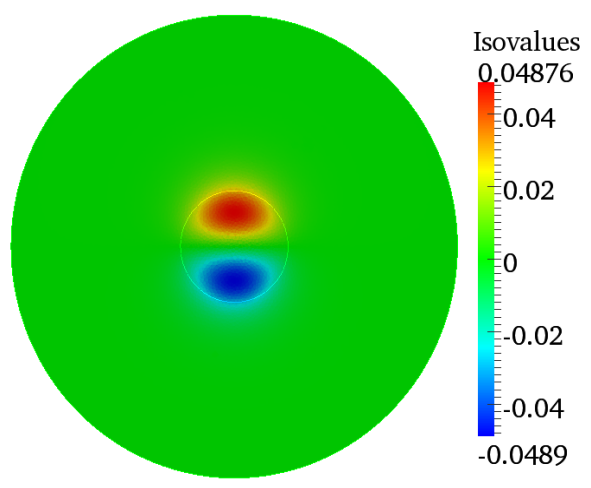

(a) Analytical

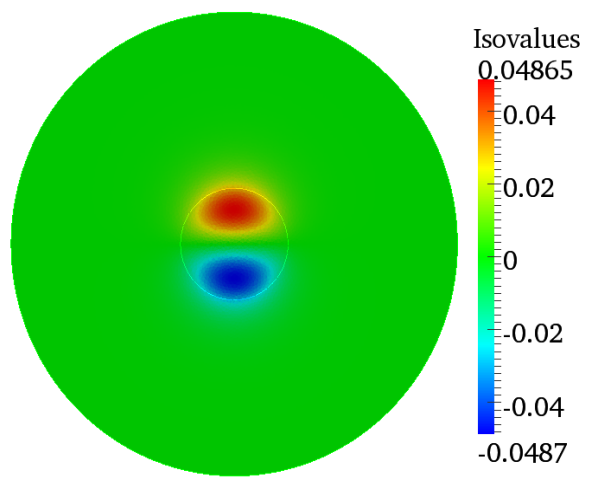

(c) Computed with $0 \%$ noise level

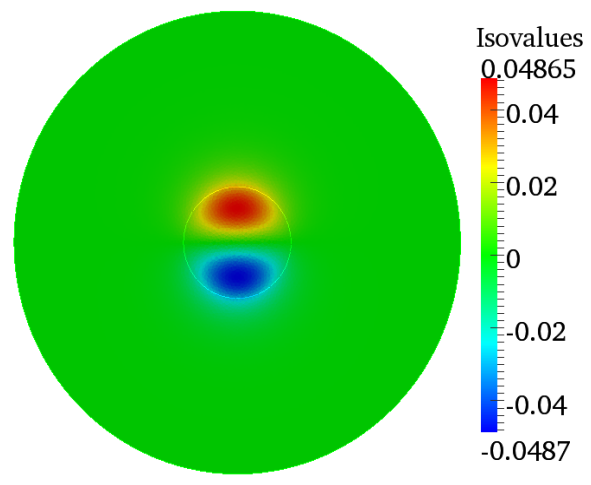

(e) Computed with $10 \%$ noise level

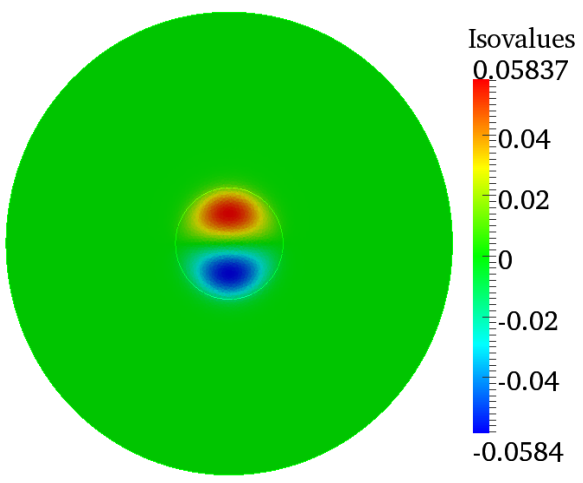

(b) Initial

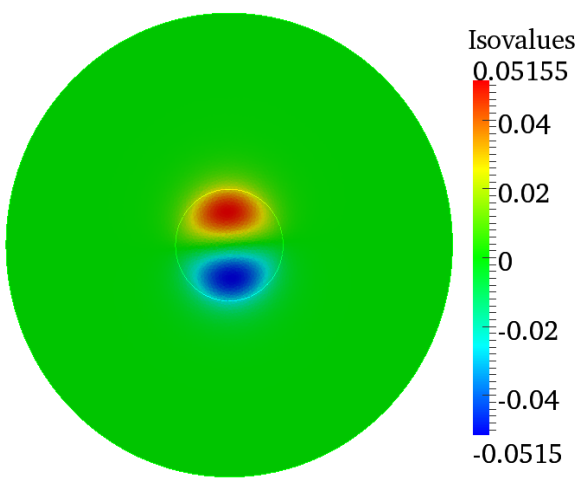

(d) Computed with $5 \%$ noise level

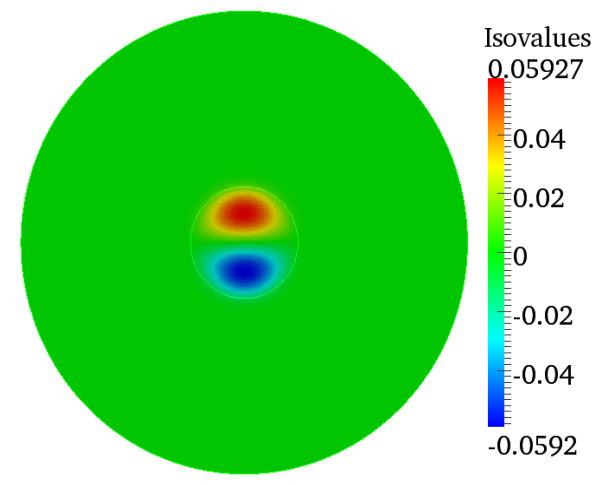

(f) Computed with $20 \%$ noise level

Figure 9: Isovalues corresponding to the guided mode $L P_{11}^{x_{2}}$. Analytic vs. computed fields for various noise levels on $\widetilde{\boldsymbol{\beta}_{2}^{\star}}$ for Experiment 2.

- Similarly to Experiment 1, the initial values of the refractive index parameters are selected outside the pre-asymptotic convergence region, since the relative error on the refractive index profile is 100\% (see Ta- 
ble 2). Moreover, this initial guess leads to the computation of propagation constants with relative residuals ranging from $67 \%$ to $100 \%$, depending on the level of noise (see equations (43)-(44)). This is also illustrated by the important difference in the values of the corresponding eigenmodes. Indeed, for example, the isovalues for the exact $L P_{11}$ field vary between -0.049 and 0.049 , whereas the ones corresponding to the initial guess range between -0.058 and 0.058 , as indicated in Figures $8(\mathrm{a})-8(\mathrm{~b})$ and Figures $9(\mathrm{a})-9(\mathrm{~b})$. Hence, this experiment is clearly performed with a "blind" initial guess value.

- Figure 7 illustrates the fast convergence and the robustness to the noise effect of the proposed solution methodology. More specifically, one can observe that the relative residual drops from the initial value of over $67 \%$, to below the noise level after one iteration only, for all considered noise levels. Furthermore, Figure 7 clearly shows that the convergence is almost monotone with almost no oscillations. This behavior is most likely due to the fact that the algorithm is applied to a two-parameter problem, and therefore, the instability effects seem to be negligible.

- At convergence, the refractive index parameters $\left(n_{+}, n_{\infty}\right)$ are delivered with an accuracy up to the noise level (see Table 3). The corresponding guided fields $\left(u_{1}, u_{2}\right)$ are also computed with a satisfactory accuracy level (see Table 3 and Figures 8-9).

\subsection{Retrieving a refractive graded-index profile}

The goal of this section is to determine the parameters corresponding to a refractive graded-index profile. To this end, we consider the class of refractive index profiles of the form:

$$
n(x)=\left\{\begin{array}{lll}
n_{+}+\alpha .|x|^{2} & ; & x \in \Omega \\
n_{\infty} & ; & x \in \Omega^{e}
\end{array}\right.
$$

and satisfying:

$$
0<n_{+}-n_{\infty} \leq 0.01 \text {. }
$$

Hence, the propagation of the modes is under the weak guidance conditions [56]-[59]. In what follows, we present the results of two numerical experiments. In the first experiment, the goal is to determine the value of the refractive index at the center of the fiber core $n_{+}$, as well as in the cladding $n_{\infty}$. In the second experiment, we recover the indices of both the core $\left(n_{+}, \alpha\right)$ and the cladding $n_{\infty}$. 


\subsubsection{Experiment 3: Partial parameters recovery}

The goal here is to retrieve two refractive index parameters, given two measured/desired eigenmodes. The unknown refractive index parameters are $n_{+}$ and $n_{\infty}$ (see equation (45) and Figure 2). Moreover, the value of the parameter $\alpha \in \mathbb{R}$ in equation (45) is chosen to ensure the continuity of the refractive graded-index profile across the interface core-cladding. Therefore, $\alpha$ is a parameter that depends on $n_{+}$and $n_{\infty}$. Consequently, we consider here a two-parameter inverse problem. The values of the target and initial refractive index profiles, $n^{\star}=\left(n_{+}^{\star}, n_{\infty}^{\star}\right)$ and $n^{(0)}=\left(n_{+}^{(0)}, n_{\infty}^{(0)}\right)$, are reported in Table 4 as well as the resulting values of $\alpha$ for both profiles. The measured guided modes corresponding to this profile are the fundamental mode $L P_{01}$ and the second mode $L P_{11}[56]-[58]$. The values of propagation constants obtained with the finite element solver [74]-[75] are given by:

$$
\boldsymbol{\beta}_{2}^{\star}=\left(\beta_{1}^{\star}, \beta_{2}^{\star}\right)^{T}=(2250.008,2249.992)^{T} .
$$

For the initial refractive index profile $n^{(0)}$ (see Table 4), its propagation constants corresponding respectively to modes $L P_{01}$ and $L P_{11}$, obtained from the dispersion equation (39), are:

$$
\boldsymbol{\beta}_{2}^{(0)}=\left(\beta_{1}^{(0)}, \beta_{2}^{(0)}\right)^{T}=(4000.005,3999.985)^{T} .
$$

We assess the sensitivity of the proposed algorithm to the noise level in $\boldsymbol{\beta}_{2}^{\star}$, by respectively adding to it three levels of white noise: $5 \%, 10 \%$ and $20 \%$, and thereafter denote the noisy $\boldsymbol{\beta}_{2}^{\star}$ by $\widetilde{\boldsymbol{\beta}_{2}^{\star}}$.

The results are reported in Table 5 and Figures 10-??. The following observations are noteworthy:

\begin{tabular}{|c||c|c||c|}
\hline $\begin{array}{c}\text { Refractive index } \\
\text { profile parameters }\end{array}$ & $n_{+}$ & $n_{\infty}$ & $\alpha$ \\
\hline \hline Target & 450.005 & 449.995 & -0.06250 \\
\hline Initial & 800.005 & 799.980 & -0.15625 \\
\hline
\end{tabular}

Table 4: Target vs. initial refractive index profile parameters for Experiment 3 . 


\begin{tabular}{|c||c|c|c|c|c|}
\hline $\begin{array}{c}\text { Initial } \\
\text { noise level } \\
\text { on } \boldsymbol{\beta}_{2}^{\star}(\%)\end{array}$ & $\begin{array}{c}\text { Relative } \\
\text { residual }(\%)\end{array}$ & $\begin{array}{c}\text { Relative } \\
\text { error } \\
\text { on } n^{\star}(\%)\end{array}$ & $\begin{array}{c}\text { Relative } \\
\text { error } \\
\text { on } \boldsymbol{\beta}_{2}^{\star}(\%)\end{array}$ & $\begin{array}{c}\text { Relative } \\
\text { error } \\
\text { on } u_{1}^{\star}(\%)\end{array}$ & $\begin{array}{c}\text { Relative } \\
\text { error } \\
\text { on } u_{2}^{\star}(\%)\end{array}$ \\
\hline \hline 0 & $1.284 \times 10^{-3}$ & $2.874 \times 10^{-3}$ & $1.284 \times 10^{-3}$ & 0.46894 & 1.68805 \\
\hline 5 & $1.124 \times 10^{-3}$ & 4.998 & 4.999 & 4.91770 & 6.81393 \\
\hline 10 & $9.397 \times 10^{-4}$ & 9.998 & 9.999 & 9.93758 & 12.49697 \\
\hline 20 & $6.587 \times 10^{-4}$ & 19.998 & 19.999 & 18.29067 & 22.12093 \\
\hline
\end{tabular}

Table 5: Sensitivity of the relative residual and the relative error, at convergence, to the noise level for Experiment 3.

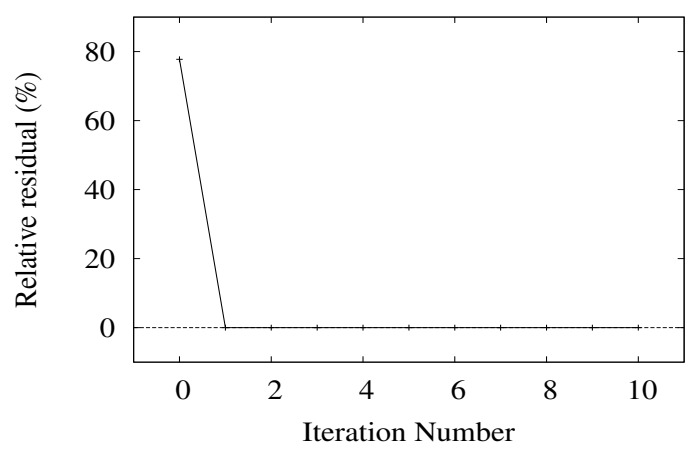

(a) Noise level on $\widetilde{\beta}=\left(\widetilde{\beta_{1}}, \widetilde{\beta_{2}}\right): 0 \%$.

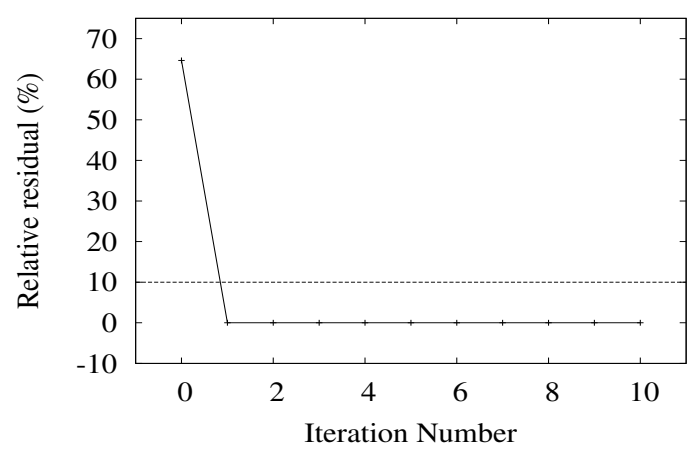

(c) Noise level on $\widetilde{\beta}=\left(\widetilde{\beta_{1}}, \widetilde{\beta_{2}}\right): 10 \%$.

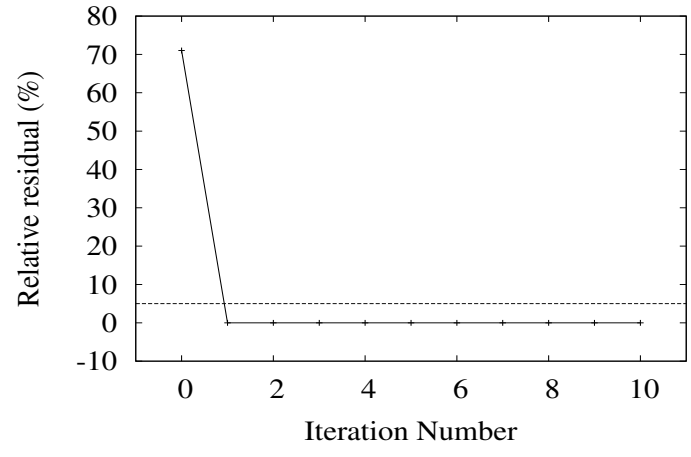

(b) Noise level on $\widetilde{\beta}=\left(\widetilde{\beta_{1}}, \widetilde{\beta_{2}}\right): 5 \%$.

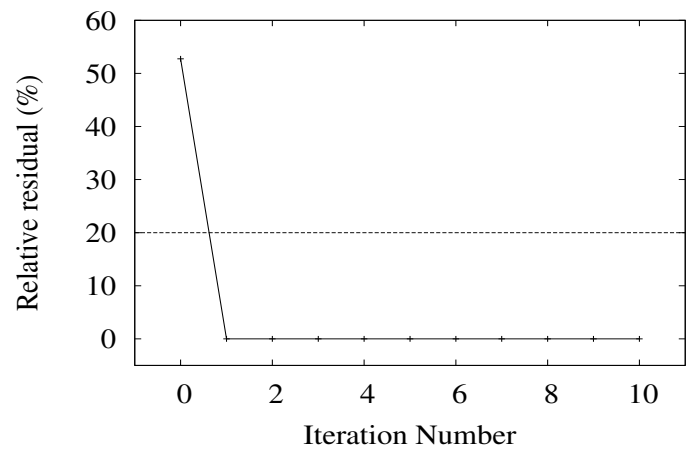

(d) Noise level on $\widetilde{\beta}=\left(\widetilde{\beta_{1}}, \widetilde{\beta_{2}}\right): 20 \%$.

Figure 10: Convergence history. Sensitivity of the relative residual given by equation (35) to the noise level on the propagation constants $\widetilde{\boldsymbol{\beta}_{2}^{\star}}$ for Experiment 3 .

- The initial values of the refractive index parameters are chosen outside the pre-asymptotic convergence region. Indeed, the initial relative 
error on the refractive index profile is $80 \%$ (see Table 4). Moreover, equations (47)-(48) indicate that this initial guess on the propagation constants results in initial relative residuals ranging from $53 \%$ to $78 \%$, depending on the level of noise. Furthermore, the isovalues of the exact $L P_{01}$ field vary between 0.0 and 0.0778 , whereas the ones corresponding to the initial guess range between 0.0 and 0.1129 . Similarly, isovalues of the exact $L P_{11}$ field vary between -0.0651 and 0.0651 , whereas the ones corresponding to the initial guess range between -0.0954 and 0.0954. Hence, this numerical experiment is performed with a "blind" initial guess value.

- Figure 10 illustrates the fast convergence and the robustness to the noise effect of the proposed solution methodology. More specifically, one can observe that the relative residual drops from the initial value of over $52 \%$, to below the noise level in one iteration only, for all considered noise levels. Furthermore, Figure 10 clearly shows that the convergence is almost monotone with virtually no oscillations.

- At convergence, the refractive index parameters $\left(n_{+}, n_{\infty}\right)$ are delivered with an accuracy up to the noise level (see Table 5). Similarly, the guided fields $\left(u_{1}, u_{2}\right)$ are delivered with an accuracy almost equal to the noise level. Clearly, the proposed method is effective in recovering the index profile and the corresponding guided modes with a satisfactory accuracy level.

\subsubsection{Experiment 4: Full parameters recovery}

The goal here is to retrieve the three refractive index parameters of the graded-index profile given by equation (45) from the knowledge of three measured/desired eigenmodes. Hence, unlike in Experiment 3, the parameter $\alpha \in \mathbb{R}$ is assumed here to be unknown and independent of $n_{+}$and $n_{\infty}$. Consequently, we apply the proposed regularized Newton algorithm to a three parameter inverse problem. The values of the target and initial refractive index profiles $n^{\star}=\left(n_{+}^{\star}, n_{\infty}^{\star}, \alpha^{\star}\right)$ and $n^{(0)}=\left(n_{+}^{(0)}, n_{\infty}^{(0)}, \alpha^{(0)}\right)$ are reported in Table 6 . The measured guided modes corresponding to the target index profile are the first three modes $L P_{01}, L P_{11}$, and $L P_{02}$ [56]-[58]. The values of the corresponding propagation constants were obtained with the finite element solver [74]-[75], and are given by:

$$
\boldsymbol{\beta}_{3}^{\star}=\left(\beta_{1}{ }^{\star}, \beta_{2}{ }^{\star}, \beta_{3}{ }^{\star}\right)^{T}=(3000.01050,2999.99609,2999.98242)^{T} .
$$

For the initial refractive index profile $n^{(0)}$ (see Table 6), the values of the corresponding propagation constants, obtained with the finite element solver [74]- 
[75], are:

$$
\boldsymbol{\beta}_{3}^{(0)}=\left(\beta_{1}{ }^{(0)}, \beta_{2}{ }^{(0)}, \beta_{3}{ }^{(0)}\right)^{T}=(5000.0068,4999.989,4999.971)^{T} .
$$

We assess the sensitivity of the proposed algorithm to the noise level in $\boldsymbol{\beta}_{3}^{\star}$, by adding to it three levels of white noise: $5 \%, 10 \%$ and $20 \%$, and thereafter denote the noisy $\boldsymbol{\beta}_{3}^{\star}$ by $\widetilde{\boldsymbol{\beta}_{3}^{\star}}$.

The obtained results are reported in Table 7 and Figures 11-12. The following observations are noteworthy:

\begin{tabular}{|c||c|c||c|}
\hline $\begin{array}{c}\text { Refractive index } \\
\text { profile parameters }\end{array}$ & $n_{+}$ & $n_{\infty}$ & $\alpha$ \\
\hline \hline Target & 600.005 & 599.995 & -0.06250 \\
\hline Initial & 1000.005 & 999.980 & -0.15625 \\
\hline
\end{tabular}

Table 6: Target vs. initial refractive index profile parameters for Experiment 4.

\begin{tabular}{|c||c|c|c|c|c|c|}
\hline $\begin{array}{c}\text { Ini. noise } \\
\text { level on } \\
\boldsymbol{\beta}_{3}^{\star}(\%)\end{array}$ & $\begin{array}{c}\text { Relative } \\
\text { residual } \\
(\%)\end{array}$ & $\begin{array}{c}\text { Relative } \\
\text { error on } \\
n^{\star}(\%)\end{array}$ & $\begin{array}{c}\text { Relative } \\
\text { error on } \\
\boldsymbol{\beta}_{3}^{\star}(\%)\end{array}$ & $\begin{array}{c}\text { Relative } \\
\text { error on } \\
u_{1}^{\star}(\%)\end{array}$ & $\begin{array}{c}\text { Relative } \\
\text { error on } \\
u_{2}^{\star}(\%)\end{array}$ & $\begin{array}{c}\text { Relative } \\
\text { error on } \\
u_{3}^{\star}(\%)\end{array}$ \\
\hline \hline 0 & $4.70 E-6$ & $1.02 E-5$ & $4.70 E-6$ & 0.383 & 1.624 & 0.723 \\
\hline 5 & $3.52 E-5$ & 5.0 & 5.0 & 2.448 & 3.211 & 3.064 \\
\hline 10 & $3.63 E-5$ & 10.0 & 10.0 & 9.223 & 9.422 & 9.695 \\
\hline 20 & 0.0 & 20.0 & 20.0 & 17.983 & 19.016 & 51.547 \\
\hline
\end{tabular}

Table 7: Sensitivity of the relative residual and the relative error, at convergence, to the noise level for Experiment 4. 


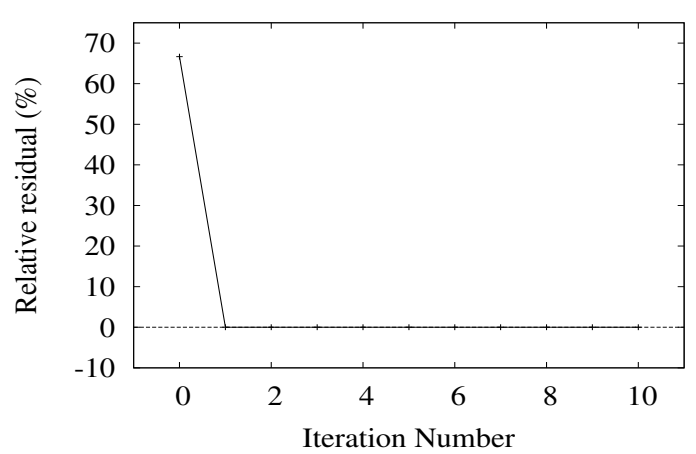

(a) Noise free

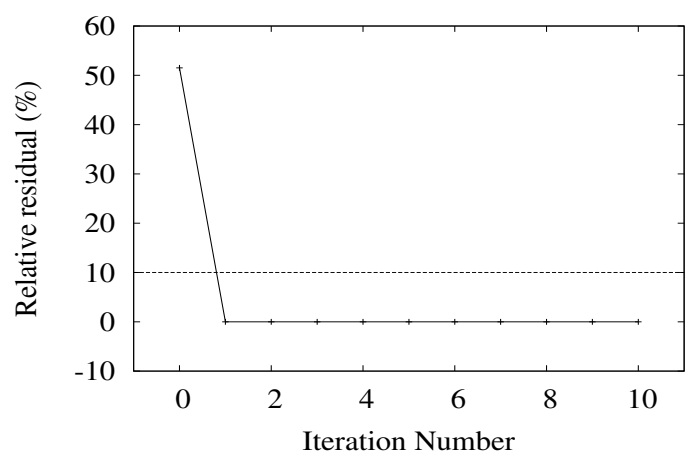

(c) Noise level $10 \%$.

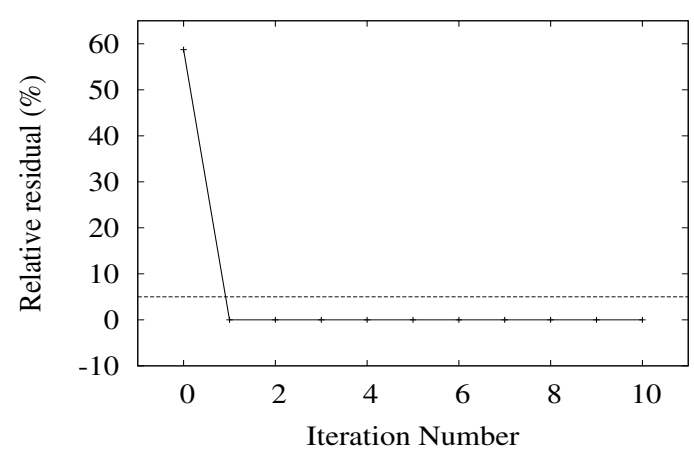

(b) Noise level $5 \%$.

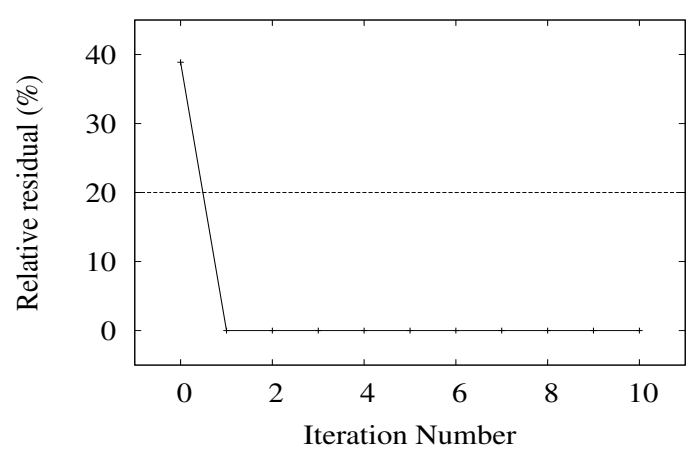

(d) Noise level $20 \%$.

Figure 11: Convergence history. Sensitivity of the relative residual given by equation (35) to the noise level on the propagation constants $\widetilde{\boldsymbol{\beta}_{3}^{\star}}$ for Experiment 4 .

- Similarly to the previous experiments, the initial values of the refractive index parameters are selected outside the pre-asymptotic convergence region. Indeed, the initial relative error on the refractive index profile parameters (see Table 6 ) is about $66.7 \%$. This initial guess leads to the computation of propagation constants with relative residuals ranging from $39 \%$ to $67 \%$, depending on the noise level (see equations (49)$(50)$ ). Moreover, the isovalues of the exact modes are significantly different from the initial ones. For example, the isovalues of the exact $L P_{02}$ field vary between -0.035 and 0.077 , whereas the ones corresponding to the initial guess range between -0.066 and 0.116 , as indicated in Figures 12(a)-12(b). Hence, this experiment is clearly performed with a "blind" initial guess value.

- Figure 11 illustrates the fast convergence and the robustness to the 


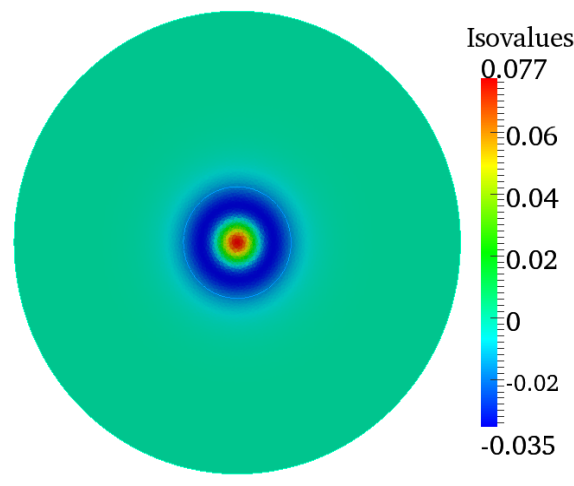

(a) Analytical

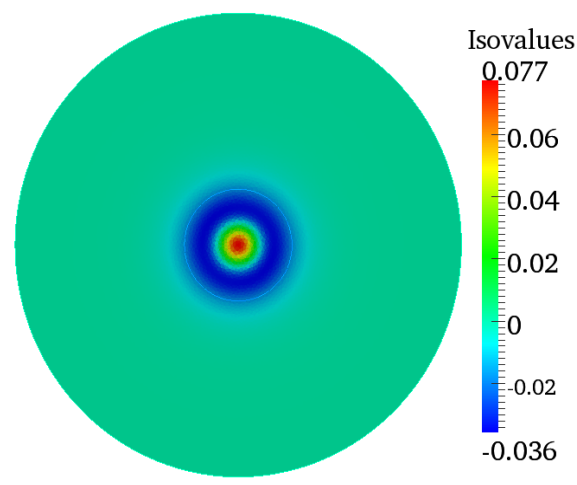

(c) Computed with $0 \%$ noise level

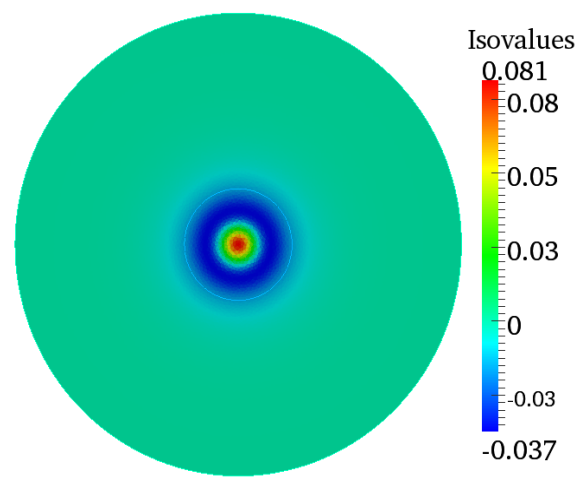

(e) Computed with $10 \%$ noise level

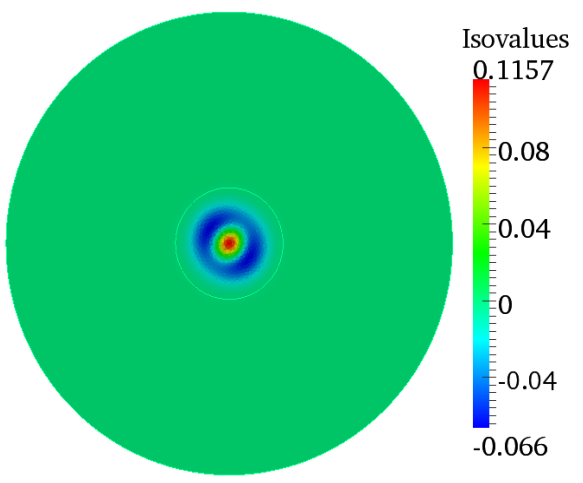

(b) Initial

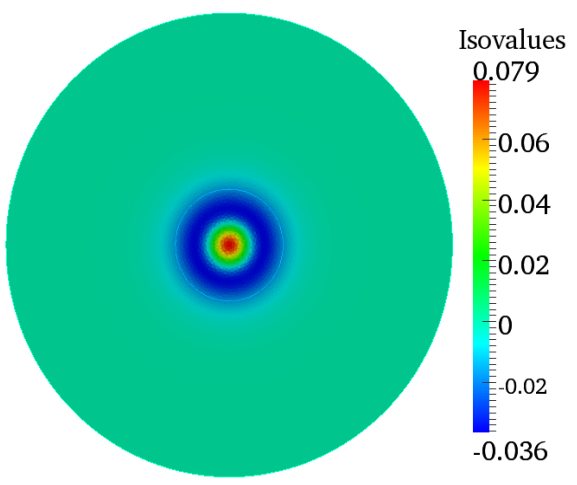

(d) Computed with 5\% noise level

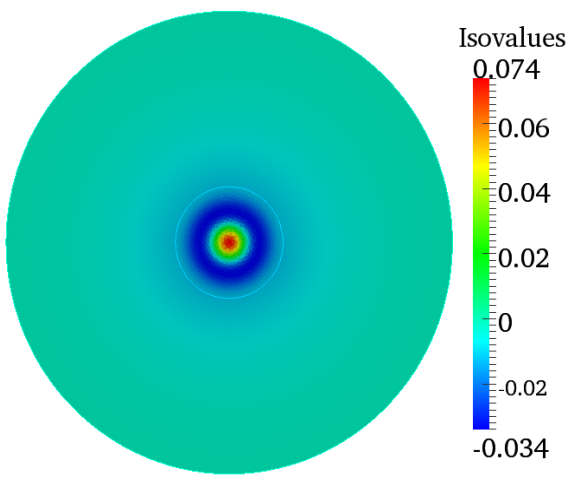

(f) Computed with $20 \%$ noise level

Figure 12: Isovalues corresponding to the guided mode $L P_{02}$. Analytic vs. computed fields for various noise levels on $\boldsymbol{\beta}_{3}^{\star}$ for Experiment 4.

noise effect of the proposed solution methodology. More specifically, one can observe that the algorithm converges after only one iteration, for all considered noise levels (see the second column in Table 7). Fur- 
thermore, Figure 11 clearly shows that the convergence is almost monotone with almost no oscillations.

- At convergence, the relative error on refractive index parameters $n^{\star}=$ $\left(n_{+}^{\star}, n_{\infty}^{\star}, \alpha^{\star}\right)$ is comparable to the noise level. Table 7 indicates the corresponding propagation constants $\boldsymbol{\beta}_{3}^{\star}$ as well as the associated eigenfunctions $u_{1}^{\star}$ and $u_{2}^{\star}$ are obtained with relative errors comparable to the noise levels. This remark is also valid for the associated eigenfunction $u_{3}^{\star}$, except when the noise level in the data is $20 \%$. This might be due to the fact that the eigenmode $L P_{02}$ is very close to the cut-off frequency [56]-[58], and therefore very sensitive to a relatively large perturbation on the value of the propagation constant $\beta_{3}^{\star}$.

\subsection{Retrieving $\mathrm{W}$-shaped refractive index profiles}

Next, we consider a third class of refractive index profiles, namely the Wrefractive index profiles, that is important to many applications [56]-[58], [60]. This class of profiles is very effective for reducing the modal dispersion effect and for enhancing the bandwidth performance of the optical fibers [60]. This class of profiles is given by the following power law [60]:

$$
n(\mathbf{x})=\left\{\begin{array}{llr}
n_{+} \sqrt{1-2 \rho \Delta\left(\frac{|\mathbf{x}|}{a}\right)^{g}} & ; & 0 \leq|\mathbf{x}| \leq a \\
n_{\infty} & ; & |\mathbf{x}| \geq a
\end{array}\right.
$$

where

- $\Delta$ is a positive number representing the refractive index difference. $\Delta$ is given by:

$$
\Delta=\frac{n_{+}^{2}-n_{\infty}^{2}}{2 n_{+}^{2}}
$$

- $g$ is a positive number, called the index component. It determines the refractive index profiles.

- $\rho$ is a positive number representing the depth of the index valley, as shown in Figure 13(a), Figure 17(a), and Figure 20(a). Observe that when $\rho=1$, the profile index given by (51) falls in the category of the graded refractive index profiles similar to the one considered in Section 5.2.

The goal here is to use the index profile parametrization given by (21) and apply the proposed algorithm to retrieve profiles given by (51) in the case 
where $\rho=1.7$ and $g=2$. The practical performance of this set of profiles has been analyzed in [60]. We investigate the sensitivity of the reconstruction to the number of parameters $N_{P}$ in (21). We present results obtained for $N_{P}=4$ and $N_{P}=5$. We also present reconstruction results with $N_{P}=3$ to demonstrate that when some a priori knowledge about the target profile is available, one can use fewer parameters.

\subsubsection{Experiment 5: Retrieving a W-refractive index profile with four parameters}

The goal here is to determine the profile depicted in Figure 13(a) from the knowledge of its first four guided modes $L P_{01}, L P_{11}, L P_{02}$, and $L P_{21}$. These synthetic four guided modes were delivered by the finite element solver introduced in Section 4.3. The corresponding propagation constants values obtained with the finite element solver [74]-[75] are:

$$
\boldsymbol{\beta}_{4}^{\star}=(7562.51270,7562.50098,7562.48877,7562.48877)^{T} .
$$

We use the parametrization given by (21) with $N_{P}=4$ and the basis functions $\left\{g_{l}\right\}_{1<l<4}$, depicted in Figure 3. Hence, we consider a four-parameter inverse problem whose unknowns are $\alpha_{1}, \alpha_{2}, \alpha_{3}$ and $\alpha_{4}$. Similarly to the previous experiments, we taint the propagation constants vector $\boldsymbol{\beta}_{4}^{\star}$ with 3 noise levels: $5 \%, 10 \%$ and $20 \%$. The initial index profile $n^{(0)}$ is a step-index profile depicted in Figure 13(b), whose parameters are: $\alpha_{1}^{(0)}=2512.505, \alpha_{2}^{(0)}=$ $\alpha_{3}^{(0)}=0$, and $\alpha_{4}^{(0)}=2512.495$. The first four propagation constants corre-

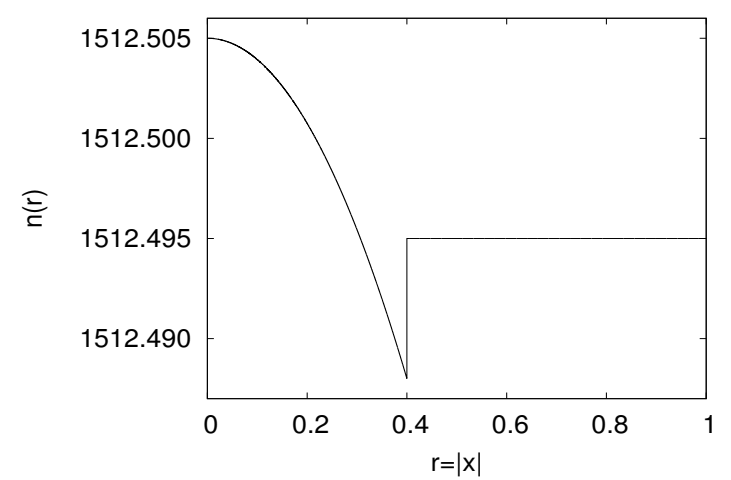

(a) Target profile: W-shape

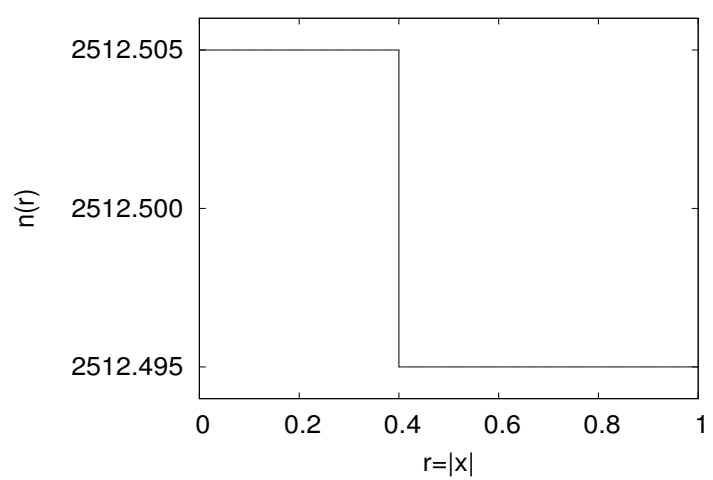

(b) Initial profile: step-index

Figure 13: Refractive index profile in Experiment 5: Target vs. initial. sponding to $n^{(0)}$ are:

$$
\boldsymbol{\beta}_{4}^{(0)}=(12562.5234,12562.5215,12562.5186,12562.5176)^{T},
$$


For each noise level in the measured propagation constants vector $\widetilde{\boldsymbol{\beta}_{4}^{\star}}$, we apply the proposed inversion algorithm from the initial step index profile $n^{(0)}$ to determine the W-refractive index depicted in Figure 13(a). The results are reported in Figures 14-16. The following observations are noteworthy:

- The algorithm is initiated outside the pre-asymptotic convergence region. Indeed, the initial refractive index profile is a step-index profile that significantly differs from the target profile which is a W-shape profile (see Figure 13). The relative error between the two profiles is over $66 \%$. Moreover, the initial relative residual on the propagation constants vector is about $67 \%$. The corresponding initial eigenfields are also very different from the ones corresponding to the $\mathrm{W}$-shape profile, as illustrated in Figures 16(a)-16(b) for the mode $L P_{21}^{x_{2}}$.

- Similarly to the previous experiments, the convergence of the proposed algorithm is relatively very fast, as demonstrated in Figure 14. We must point out that in this case the regularization was critical to ensure the convergence of the algorithm.

- Figure 15 shows that, at convergence, the sought-after refractive index profile is delivered with an accuracy ranging from $10^{-5} \%$ (in the absence of noise) to about $20 \%$ when the noise level is $20 \%$. The recovery of the W-shape profile appears to be quite satisfactory, as illustrated in Figure 16 in which the mode $L P_{21}^{x_{2}}$ is depicted. The relative error varies from $4 \%$ to $18 \%$, depending on the noise level. 


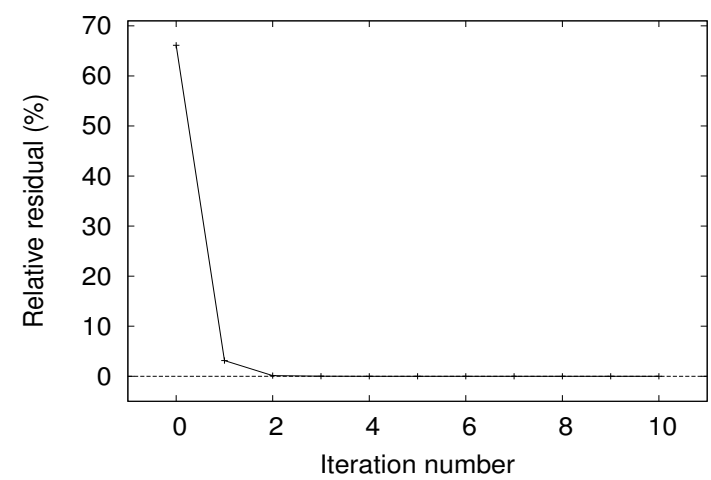

(a) Noise free

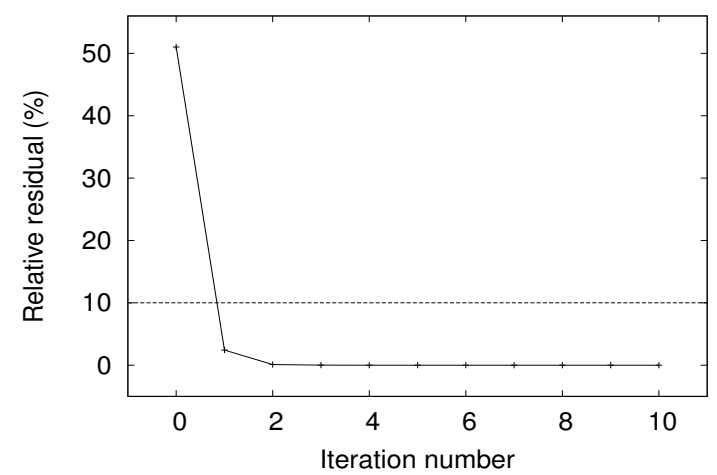

(c) Noise level $10 \%$.

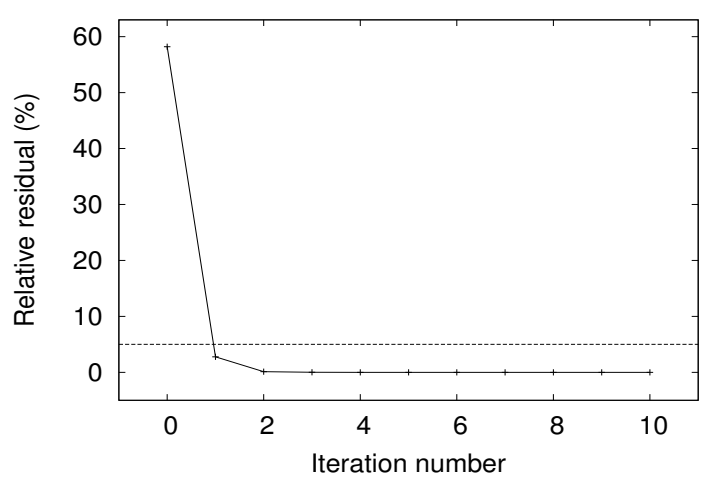

(b) Noise level $5 \%$.

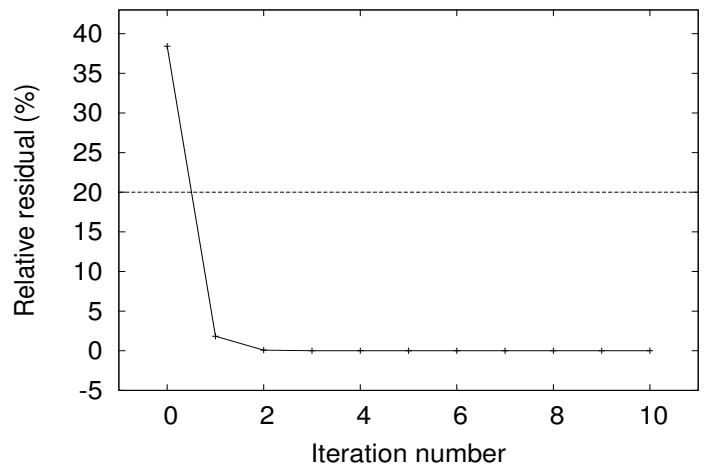

(d) Noise level $20 \%$.

Figure 14: Convergence history. Sensitivity of the relative residual given by equation (35) to the noise level on the propagation constants $\widetilde{\boldsymbol{\beta}_{4}^{\star}}$ for Experiment 5 . 


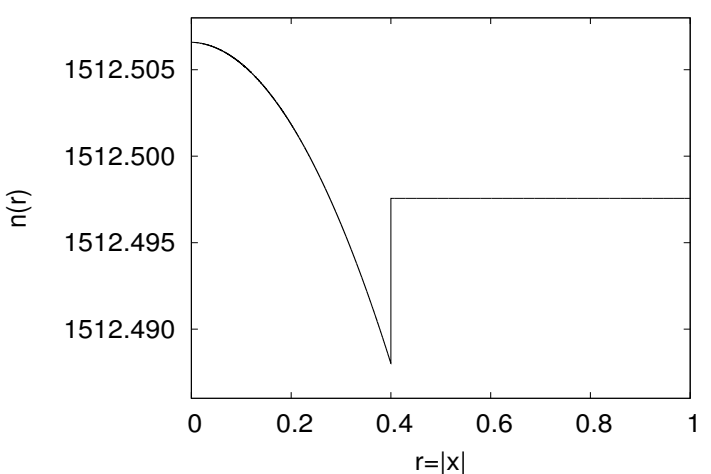

(a) Noise level $0 \%$.

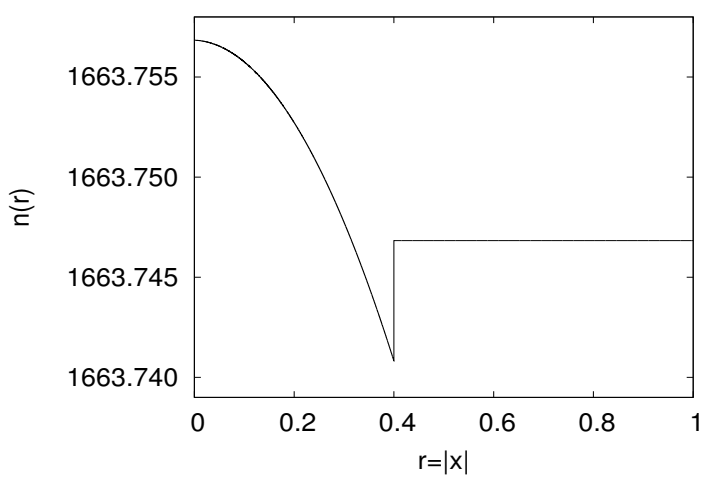

(c) Noise level $10 \%$.

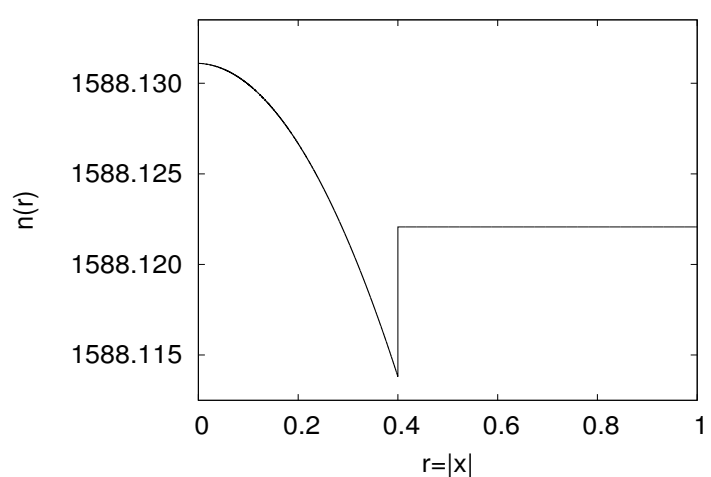

(b) Noise level $5 \%$.

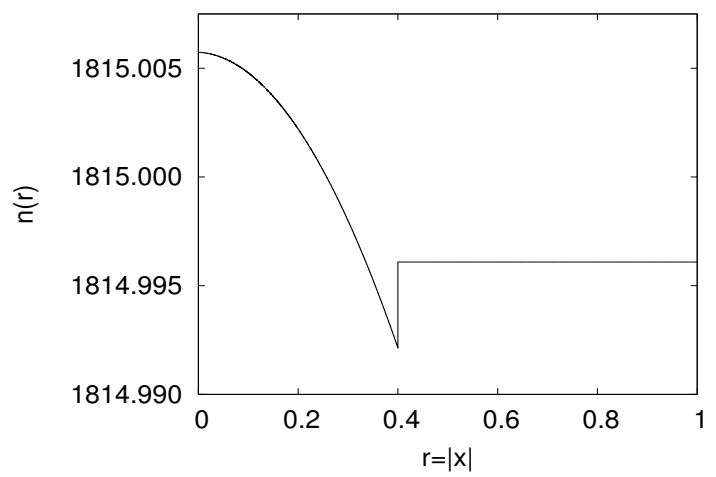

(d) Noise level $20 \%$.

Figure 15: Sensitivity of computed refractive index profile at convergence to the noise level on the propagation constants $\widetilde{\boldsymbol{\beta}_{4}^{\star}}$ in Experiment 5 . 


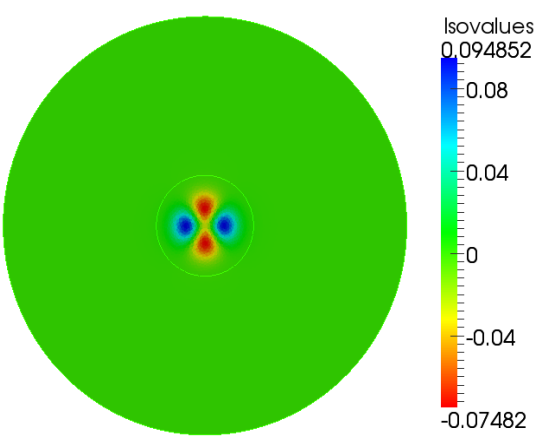

(a) Target

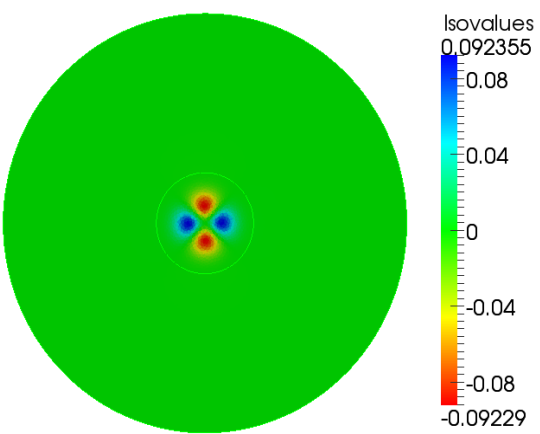

(c) Computed with $0 \%$ noise level

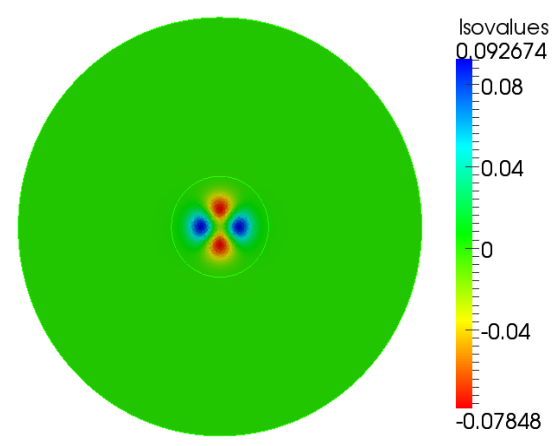

(e) Computed with $10 \%$ noise level

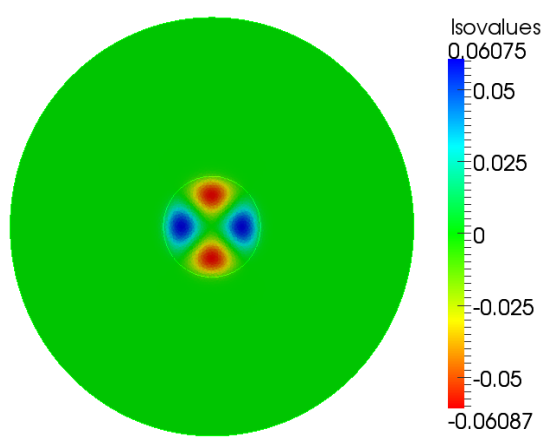

(b) Initial

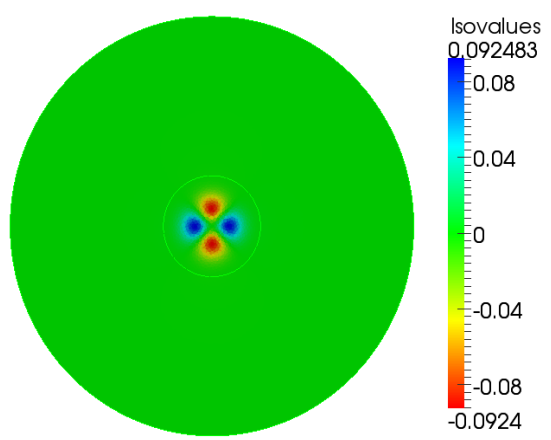

(d) Computed with $5 \%$ noise level

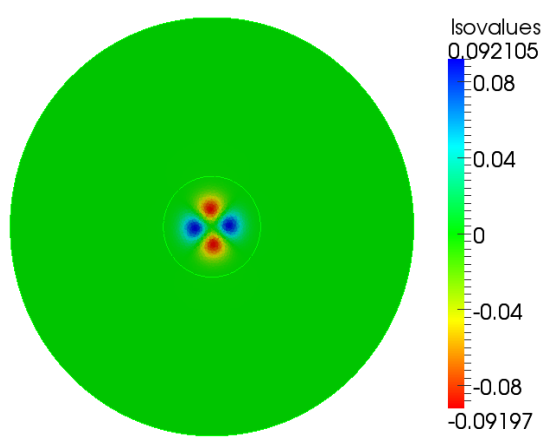

(f) Computed with $20 \%$ noise level

Figure 16: Isovalues corresponding to the guided mode $L P_{21}^{x_{2}}$. Target vs. computed fields for various noise levels on $\widetilde{\boldsymbol{\beta}_{4}^{\star}}$ for Experiment 5 .

\subsubsection{Experiment 6: Retrieving a W-refractive index profile with five parameters}

The main objective in this experiment is to investigate the effect of increasing the number of parameters in the approximation given by (21) on the per- 
formance (convergence and accuracy) of the proposed inversion algorithm. To this end, we consider a vector of five measured propagation constants corresponding to the modes $L P_{01}, L P_{11}, L P_{02}, L P_{21}, L P_{12}$. The synthetic vector $\boldsymbol{\beta}_{5}^{\star}$ computed with the finite element solver [74]-[75], is given by:

$$
\boldsymbol{\beta}_{5}^{\star}=(9000.01367,9000.00293,8999.99219,8999.99219,8999.98145)^{T}
$$

and corresponding to the sought-after W-refractive index profile depicted in Figure $17(\mathrm{a})$. We use the parametrization given by (21) with $N_{P}=5$. Note that we also employ the basis functions depicted in Figure 3, but with $g_{4}$ being the polynomial function of degree 3 extended by 0 for $r \geq a$, and $g_{5}$ being the function depicted in Figure 3(d). We thus consider a five-parameter inverse problem whose unknowns are $\alpha_{j}, j=1, \cdots, 5$. Similarly to Experiment 5, measurements are contaminated with the same noise levels. The initial index profile $n^{(0)}$ is also a step-index profile (see Figure 17(b)) whose first five propagation constants, obtained with the finite element solver [74]-[75], are:

$\boldsymbol{\beta}_{5}^{(0)}=(14250.0234,14250.0215,14250.0195,14250.0186,14250.0166)^{T}$

For each noise level in the measured propagation constants vector $\widetilde{\boldsymbol{\beta}_{5}^{\star}}$, we apply the proposed algorithm from the initial step-index profile $n^{(0)}$ to determine the W-refractive index profile in Figure 17(a). The results are reported in Figures 18-??. These results reveal the following:

- Similarly to Experiment 5, the algorithm is initiated from a refractive index profile (a step index) that significantly differs from the target profile (W-shape), as indicated in Figure 17. The initial relative error is about $60 \%$, resulting in an initial relative residual of also about $60 \%$. Clearly, the inversion algorithm is initiated outside the pre-asymptotic region.

- Figure 18 shows that the convergence of the algorithm is comparable to the one observed in Experiment 5 when using only 4 parameters. In addition, the algorithm does not converge without incorporating the regularization procedure.

- The sought-after refractive index profile is determined with an accuracy level comparable to the case of 4 parameters. Indeed, at convergence, the relative error on the refractive index profile ranges from $10^{-5} \%$ (in the absence of noise) to about $20 \%$ when the noise level is $20 \%$. On the other hand, we observed an improvement in the accuracy of the corresponding eigenmodes. For example, the highest mode $L P_{12}$ is 


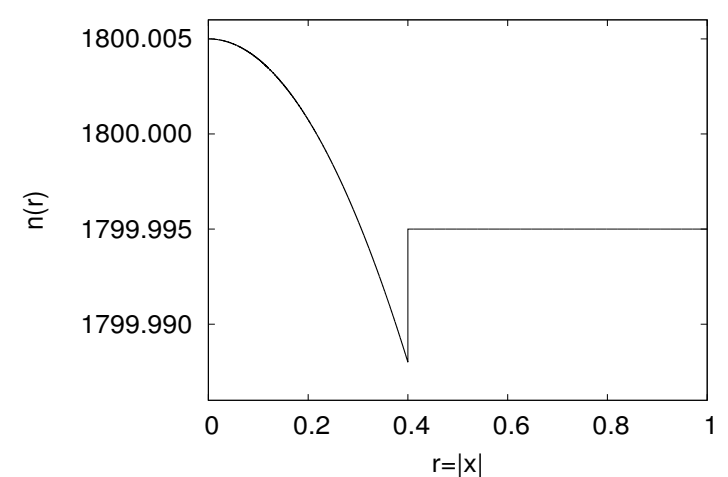

(a) Target profile: W-shape

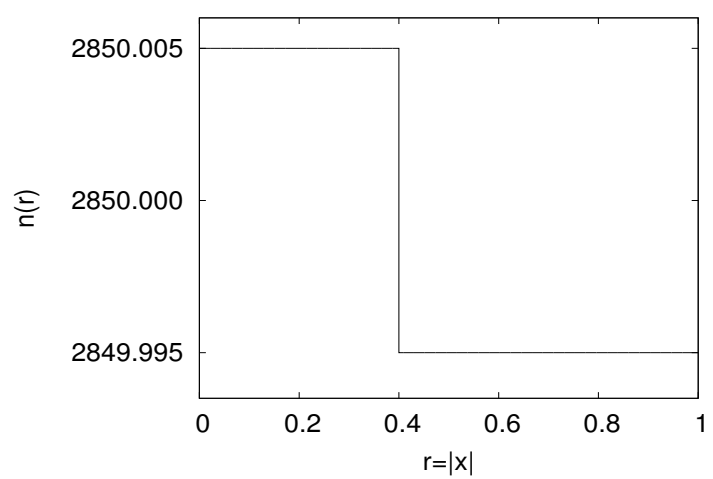

(b) Initial profile: step-index

Figure 17: Refractive index profile in Experiment 6: Target vs. initial.

computed with a relative error ranging from $8.5 \%$ to $11 \%$ depending on the noise level. 


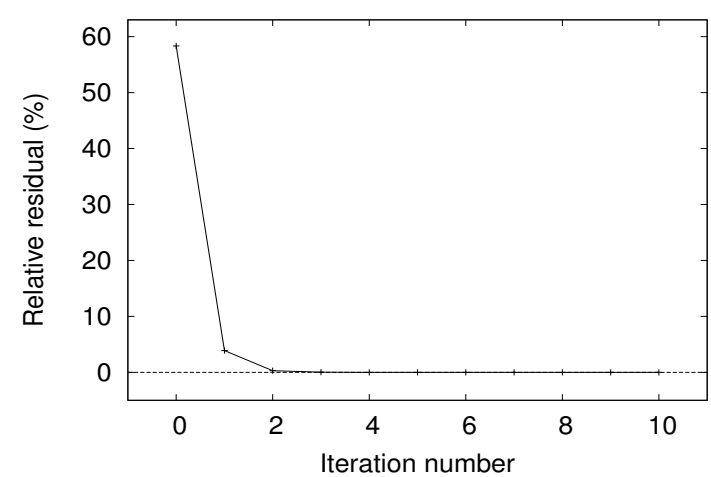

(a) Noise free

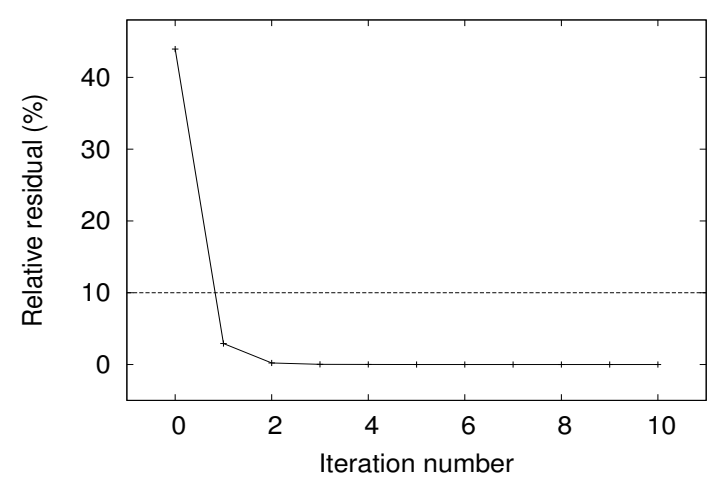

(c) Noise level $10 \%$.

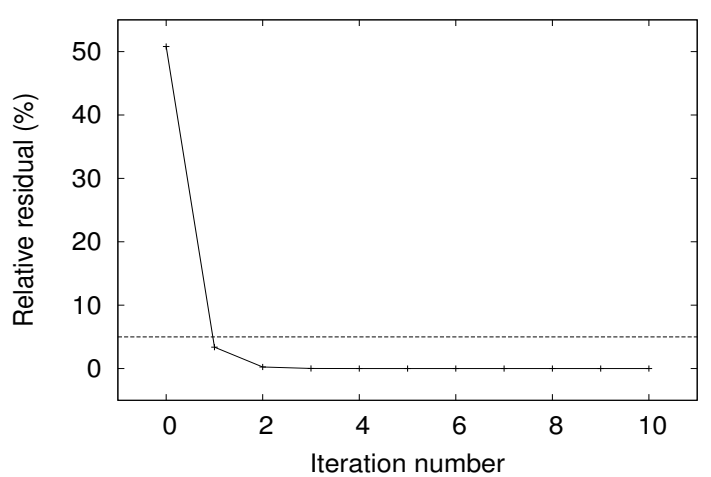

(b) Noise level $5 \%$.

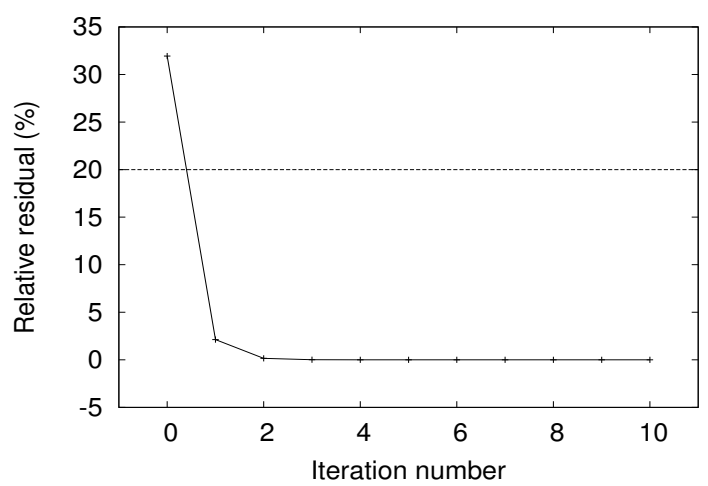

(d) Noise level $20 \%$.

Figure 18: Convergence history. Sensitivity of the relative residual given by equation (35) to the noise level on the propagation constants $\widetilde{\boldsymbol{\beta}_{5}^{\star}}$ for Experiment 6 .

\subsubsection{Experiment 7: Retrieving a W-refractive index profile with three parameters}

The goal of this experiment is to demonstrate that when some a priori knowledge on the sought-after profile is available, it is possible to successfully recover the profile using fewer parameters in (21). For this numerical experiment, The target profile (W-refractive) is depicted in Figure 20(a). We employ the parametrization given by (21) with $N_{P}=3$. Note that the $g_{1}$ is the constant depicted in Figure $3(\mathrm{a}), g_{2}$ is the quadratic polynomial function depicted in Figure 3(c), and $g_{3}$ is the constant function depicted in Figure 3(d). Hence, we assume a priori that this set of trial solutions can describe the sought-after W-profile. The synthetic measurements are the first guided modes $L P_{01}, L P_{11}$, and $L P_{02}$ whose propagation constants are:

$$
\boldsymbol{\beta}_{3}^{\star}=(5062.51025,5062.49561,5062.48145)^{T} .
$$




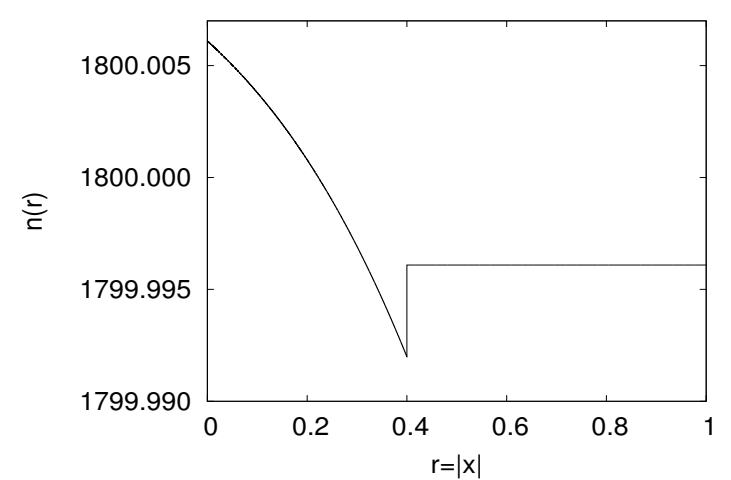

(a) Noise level $0 \%$.

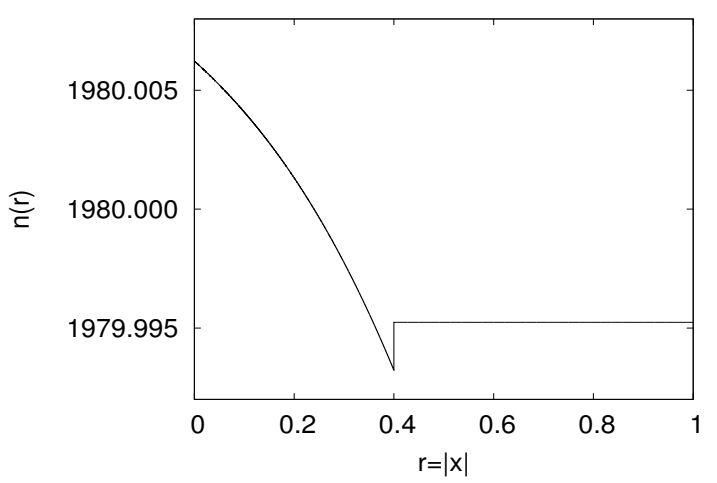

(c) Noise level $10 \%$.

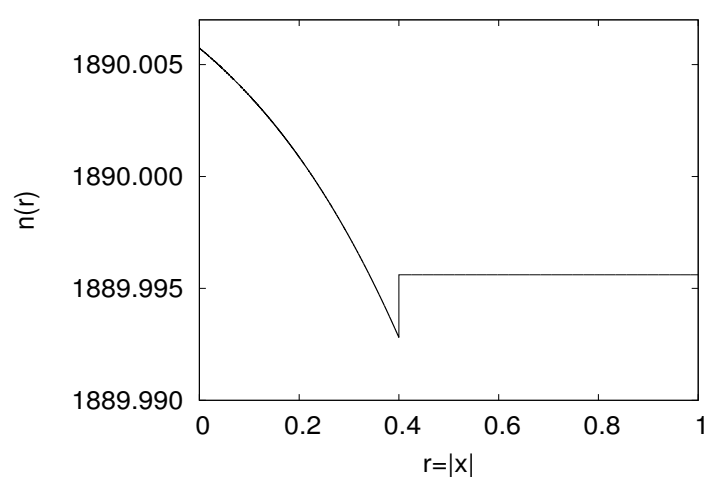

(b) Noise level $5 \%$.

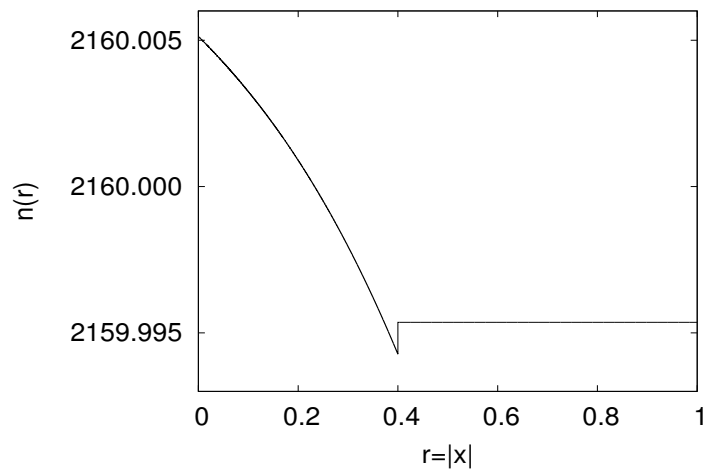

(d) Noise level $20 \%$.

Figure 19: Sensitivity of computed refractive index profile at convergence to the noise level on the propagation constants $\widetilde{\boldsymbol{\beta}_{5}^{\star}}$.

The initial index profile $n^{(0)}$ is this time chosen to be a guided-index profile depicted in Figure 20(b), whose first three propagation constants are:

$$
\boldsymbol{\beta}_{3}^{(0)}=(10062.5176,10062.5107,10062.5039)^{T} .
$$

Similarly to all previous experiments, the synthetic propagation constants are tainted with white noise of the same three levels. For each noise level, we apply the proposed inversion algorithm from the initial profile $n^{(0)}$ to determine the $\mathrm{W}$-refractive index profile in Figure 20(a). The results are reported in Figures 21-22. The results of this experiment suggest the following:

- Even though the algorithm is starting from an "educated" guess $n^{(0)}$, the initial relative error on the refractive index profile is about $100 \%$ and the initial relative residual is also about $100 \%$. This means that the inversion algorithm is still initiated outside the pre-asymptotic convergence region. 


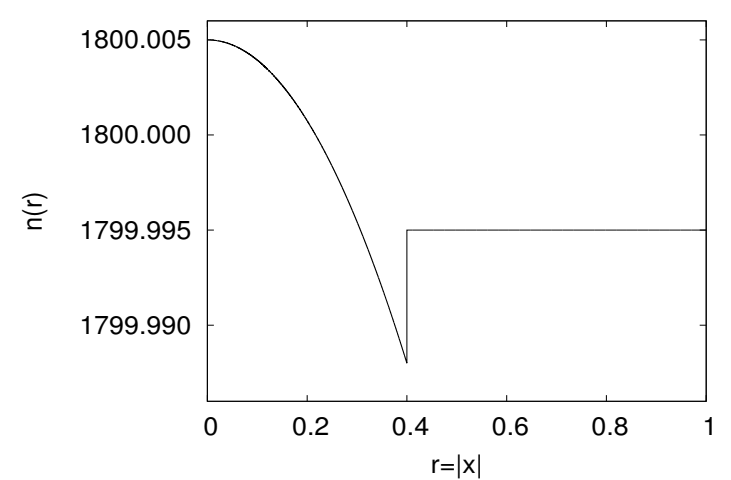

(a) Target profile

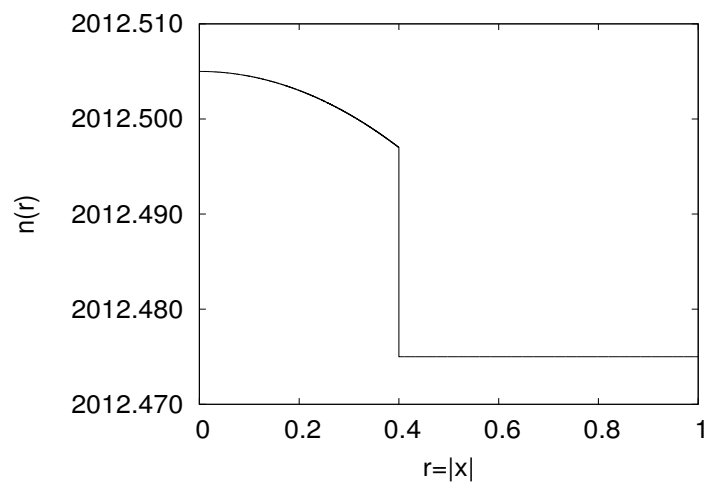

(b) Initial profile

Figure 20: Refractive index profile in Experiment 7: Target vs. initial.

- Figure 21 indicates that the inversion algorithm converges in less than three iterations, regardless of the noise level. At convergence, the algorithm delivers refractive index profiles with a high accuracy level as depicted in Figure 22. Indeed, the relative error ranges from $10^{-5} \%$ (for $0 \%$ noise level) to $20 \%$ (for $20 \%$ noise level).

Remark. It is worth mentioning that the proposed solution methodology fails to retrieve the target refractive index profile in the following two situations:

- when the number of measured/desired guided modes is smaller than the number of the target refractive index profile parameters. These cases require solving at each Newton iteration under-determined parameters problems. We have observed that the proposed computational procedure does not converge even for simple situations such as refractive step-index profiles with initial guess values very close to the target values.

- when the target refractive index profile cannot be described by the shape parametrization adopted for representing the trial solutions, i.e., the selected parametrization is incomplete. This has been observed when the target profile is a W-refractive index profile and the selected parametrization employs basis functions $g_{1}$ and $g_{2}$ depicted in Figure 2 .

\section{Summary and Conclusion}

We have investigated mathematically and numerically the important problem of determining refractive index profiles that accommodate a measured/desired 


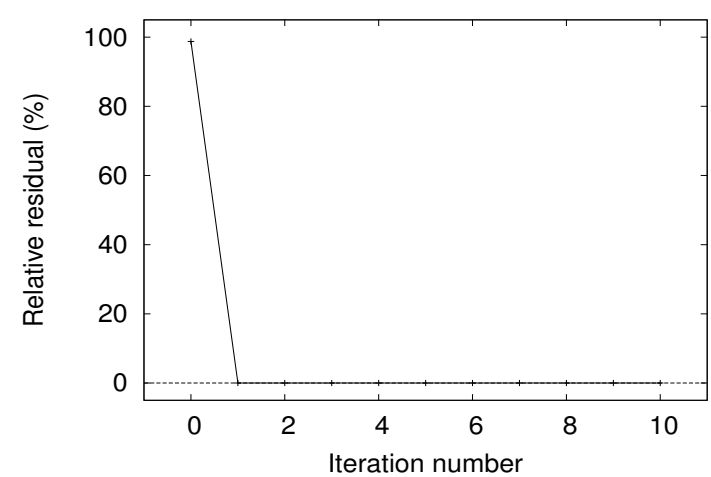

(a) Noise free

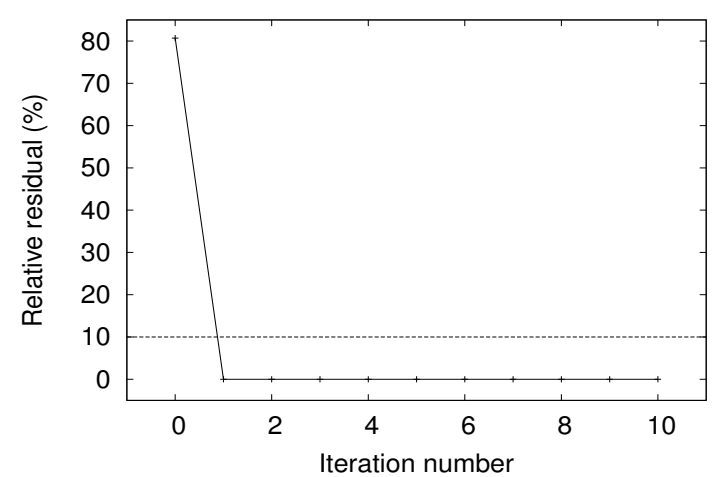

(c) Noise level $10 \%$.

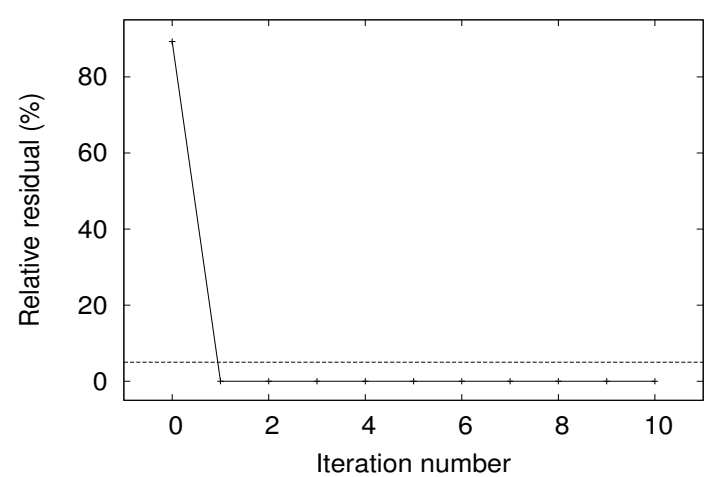

(b) Noise level $5 \%$.

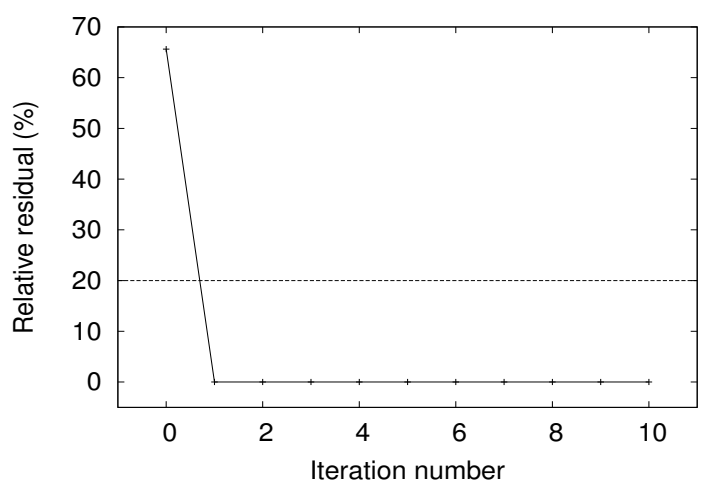

(d) Noise level $20 \%$.

Figure 21: Convergence history. Sensitivity of the relative residual given by equation (35) to the noise level on the propagation constants $\widetilde{\boldsymbol{\beta}_{3}^{\star}}$ for Experiment 7 .

guided mode propagation in homogeneous optical fibers under the weak guidance conditions. This nonlinear and ill-posed inverse problem falls in the category of inverse spectral problems that consists of finding the potential of a scalar elliptic operator from the partial knowledge of its discrete spectrum. From a mathematical view point, we have established the uniqueness of the refractive index profile from the knowledge of only one guided mode, i.e., the knowledge of one eigenvalue and its corresponding eigenfunction is enough to uniquely determine the refractive index profile. We have also provided a characterization of the derivative of the guided modes with respect to the refractive index profile. This result is crucial for an accurate computation of the Jacobians occuring at the Newton iteration equations.

From a numerical point of view, we have proposed a regularized iterative method to compute the refractive index profile parameters when some guided modes are given. Numerical experiments were performed to retrieve three 


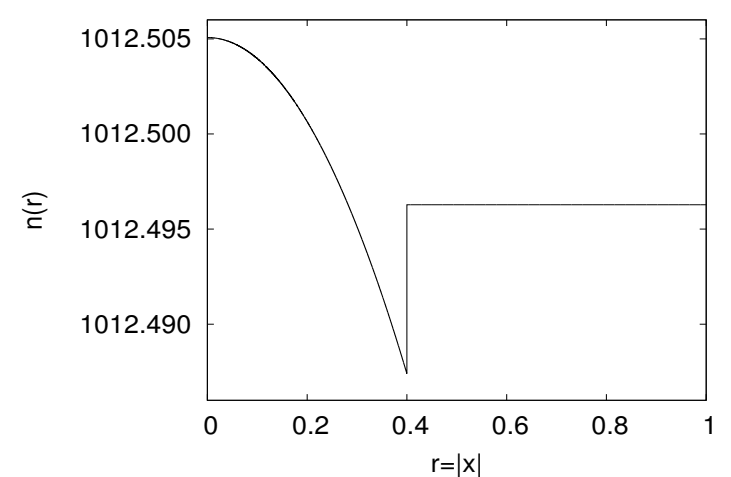

(a) Noise level $0 \%$.

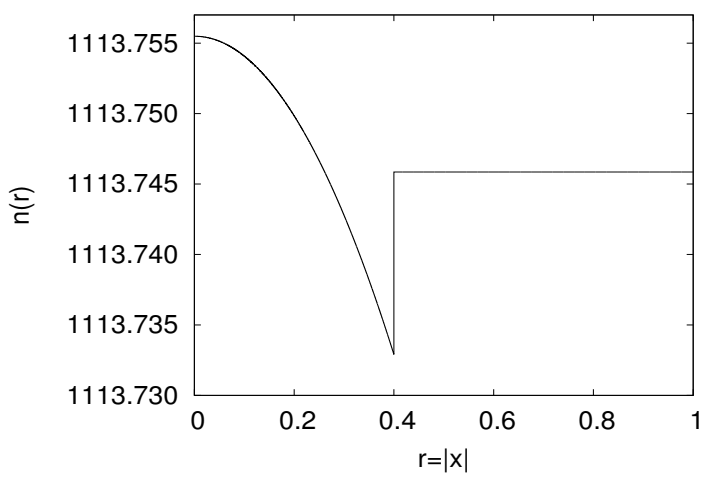

(c) Noise level $10 \%$.

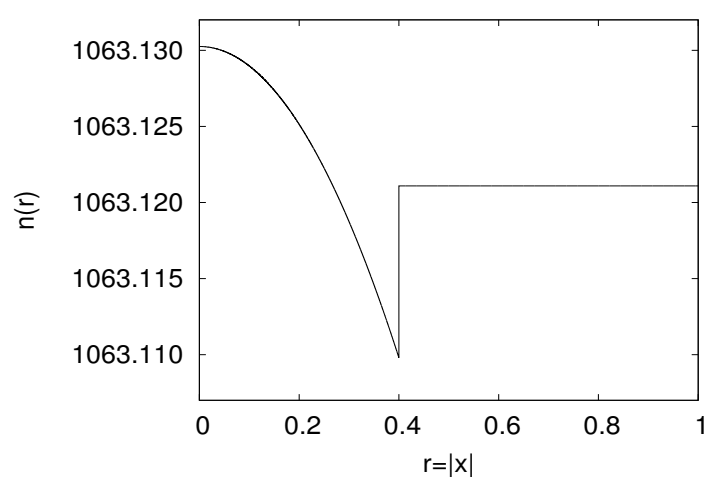

(b) Noise level $5 \%$.

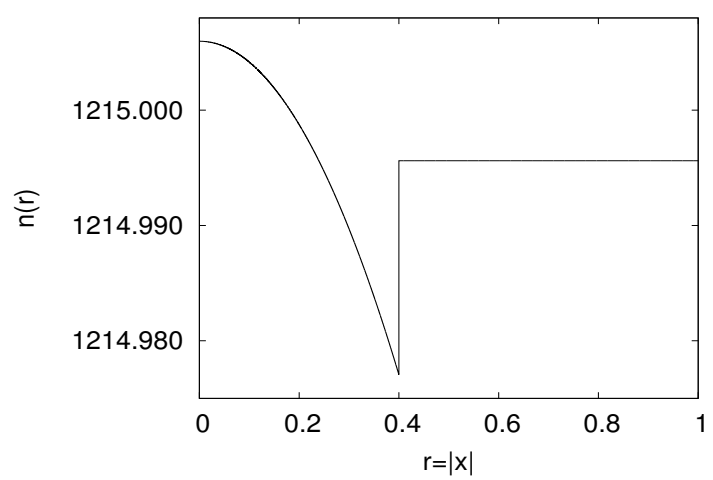

(d) Noise level $20 \%$.

Figure 22: Sensitivity of computed refractive index profile at convergence to the noise level on the propagation constants $\widetilde{\boldsymbol{\beta}_{3}^{\star}}$.

classes of refractive index profiles: the step-index, the graded-index, and the W-shape. The obtained results demonstrate that the proposed computational procedure is accurate, effective, and robust to the noise. Indeed, in all numerical experiments, the refractive index profiles are accurately retrieved up to the noise level after few iterations.

Acknowledgment. The authors acknowledge the support of the European Union's Horizon 2020 research and innovation program under the Marie Sklodowska Curie grant agreement $N^{o}$ 644602. They also thank the anonymous referees for their constructive remarks and suggestions. Any opinions, findings, conclusions or recommendations expressed in this material are those of the authors and do not necessarily reflect the views of CSUN or INRIA. 


\section{References}

[1] Protter M. H. Can one hear the shape of a drum? Revisted. Siam Review 29 (2), (1987), pp. 185-197.

[2] Bérard P. H. Spectral geometry: direct and inverse problems. Lecture Notes in Mathematics 1207, Springer-Verlag, New-York, 1986.

[3] Osgood B., Phillips R., and Sarnak P. Compact isospectral sets of surfaces. J. Funct. Analysis 80, (1988), pp. 212-234.

[4] Guillemin V. Inverse spectral results on two-dimensional tor. J. of AMS 3, (1990), pp. 375-387.

[5] Allaire G.,Aubry S., and Jouve F. Eigenfrequency optimization in optimal design. Comput. Methods Appl. Mech. Engrg. 190, (2001), pp. 3565-3579.

[6] Isakov V. Inverse problems for partial differential equations. Springer, New-York, 1998.

[7] Borg G. Eine umkehrung der Sturm-Liouvilleschen eigenwertaufgabe. Acta Math. 78, (1946), pp. 1-96.

[8] Levinson N. The inverse Sturm-Liouville problem. Mat. Tidsskr. B, (1949), pp. 25-30.

[9] Marchenko V. M. Concerning the theory of a differential operator of the second order. Dokl. Akad. Nauk SSSR 72, (1950), pp. 457-460.

[10] Hoschstadt H. The inverse Sturm-Liouville problem. Comm. Pure Appl. Math. 26, (1973), pp. 715-729.

[11] Hoschstadt H. Well-posed inverse spectral problems. Proc. Nat. Acad. Sci. 72, USA, (1975), pp. 2496-2497.

[12] Krein M. G. Solution of the inverse Sturm-Liouville problem. Dokl. Akad. Nauk SSSR 76, (1951), pp. 21-24.

[13] Gelfand I. M. and Levitan B. M. On the determination of a differential equation from its spectral function. Amer. Math. Soc. Transl. 1, (1955), pp. 253-304.

[14] Levitan B. M. On the determination of a Sturm-Liouville equation by two spectra. Amer. Math. Soc. Transl. 68, (1968), pp. 1-20. 
[15] Niordson F. I. A method for solving inverse eigenvalue problems, in: Recent Progress in Applied Mechanics. The Folke Odquist Volume (Broberg B., Hult J. \& Niordson F., Editors), Almquist \& Wiksell, Stockholm, (1967), pp. 375-382.

[16] Hald O. H. On the discrete and numerical inverse Sturm-Liouville problems. Ph.D. thesis, New-York University, New-York, 1972.

[17] Barcilon V. Iterative solution of the inverse Sturm-Liouville problem. J. Mathematical Phys. 15, (1974), pp. 287-298.

[18] Friedland S. Inverse eigenvalue problems. Linear Algebra and Appli. 17, (1977), pp. $15-51$.

[19] Marchenko V. A. Sturm-Liouville operators and applications. Birkhäuser, 1986.

[20] Pöschel J. and Trubowitz E. Inverse spectral theory Academic Press, Orlando, 1987.

[21] Coleman C. F. and McLaughlin J. R. Solution of the inverse spectral problem for an impedance with integrable derivative, Part I. Comm. Pure Appl. Math. 46, (1993), pp. 145-184.

[22] Coleman C. F. and McLaughlin J. R. Solution of the inverse spectral problem for an impedance with integrable derivative, Part II. Comm. Pure Appl. Math. 46, (1993), pp. 185-212.

[23] Eskin G. and Ralston J. On isospectral periodic potentials in $\mathbb{R}^{n}$, I. Comm. Pure Appl. Math. 37, (1984), pp. 647-676.

[24] Eskin G. and Ralston J. On isospectral periodic potentials in $\mathbb{R}^{n}$, II. Comm. Pure Appl. Math. 37, (1984), pp. 715-753.

[25] DeTurck D. and Gordon C. Isospectral deformations, I. Riemann structures on two-step nil spaces. Comm. Pure Appl. Math. 40, (1987), pp. $367-387$.

[26] DeTurck D. and Gordon C. Isospectral deformations, II. Trace formulas, metrics, and potentials. Comm. Pure Appl. Math. 42, (1989), pp. 10671095 .

[27] Simon B. A new approach to inverse spectral theory, I. Fundamental formalism. Ann. of math. 150, (1999), pp. 1029-1057. 
[28] Simon B. and Gesztesy F. A. A new approach to inverse spectral theory, II. General real potentials and the connection to the spectral measure. Ann. of math. 152, (2000), pp. 593-643.

[29] Simon B. and Ramm A. A new approach to inverse spectral theory, II. Short range potentials. J. Anal. Math. 80, (2000), pp. 319-334.

[30] McLaughlin J. R. Formulas for finding coefficients from nodes/nodal lines. Proceedings of the International Congress of Mathematicians, Zürich, Switzerland, Birkhäuser, (1995), pp. 1494-1501.

[31] Hald O. H. and McLaughlin J. R. Inverse nodal problems; finding the potential from nodal lines. AMS Memoire, January, 1996.

[32] Lee C. J. and McLaughlin J. R. Finding the density for a membrane from nodal lines. In: Inverse Problems in Wave Propagation, (Chavent G., Papanicolaou G., Sacks P., and Symes W. W., Editors), SpringerVerlag, (1997), pp. 325-345.

[33] McLaughlin J. R. and Portnoy A. Perturbation expansions for eigenvalues and eigenvectors for a rectangular membrane subject to a restorative force. Comm. P.D.E 23, (1998), pp. 243-285.

[34] Eskin G., Ralston J. V., and Trubowitz E. The multidimensional inverse spectral problem with a periodic potential. Contemp. Math. 27, AMS, Providence, RI, 1984.

[35] Avdonin S. A. and Mikhaylov V. S. The boundary control approach to inverse spectral theory. Inverse Problems 26 (4), 2010.

[36] Hryniv, R. and Pronska, N. Inverse spectral problems for energydependent Sturm-Liouville equations. Inverse Problems 28 (8), 2012.

[37] Avdonin S. A., Mikhaylov, V. S., and Ramdani, K. Reconstructing the potential for the one-dimensional Schrödinger equation from boundary measurements. IMA Journal of Mathematical Control and Information 31 (1), (2014), pp. 137-150.

[38] Eckhardt J., Gesztesy F., Nichols R., and Teschl G. Inverse spectral theory for Sturm-Liouville operators with distributional potentials. J. London Math. Soc. 88 (3), (2013), pp. 801-828.

[39] Colton D. and Leung Y. J. Complex eigenvalues and the inverse spectral problem for transmission eigenvalues. Inverse Problems 29 (10), (2013), pp. 253-259. 
[40] Colton D., Leung Y. J., and Meng S. The inverse spectral problem for exterior transmission eigenvalues. Inverse Problems 30 (5), 2014.

[41] Kavian O., Kian Y., and Soccorsi E. Uniqueness and stability results for an inverse spectral problem in a periodic waveguide. Journal de Mathématiques Pures et Appliquées 104 (6), (2015), pp.1160-1189.

[42] Horváth M. and Sáfár O. Inverse eigenvalue problems. Journal of Mathematical Physics 57 (11), 2016.

[43] Cakoni F, Colton D., and Haddar H. Inverse scattering theory and transmission eigenvalues. SIAM, 2016.

[44] Khomchenko A. V. Waveguide Spectroscopy of thin Films. Academic Press, New-York, 2005.

[45] Michtchenko A. and Parra G. C. Reconstruction of Refractive Index Profile of Optical Fibers by Near Field Technique. IEEE, $3^{\text {rd }}$ International Conference on Electrical and Electronics Engineering, 2006.

[46] Boucouvalas A. C. and Thraskias C.A. Accurate Optical Fiber Refractive Index Reconstruction From Near Field. $6^{\text {th }}$ International Symposium on Communication Systems, Networks and Digital Signal Processing, Graz, Austria, pp. 500-505, 2008.

[47] Beilina L. and Klibanov M. V. Approximate global convergence and adaptivity for coefficient inverse problems. Springer, New-York, 2012.

[48] Frolov A. and Kartchevskiy E. Integral Equation Methods in Optical Waveguide Theory. Springer Proceedings in Mathematics and Statistics, Vol. 52, 2013, pp. 119-133.

[49] Spiridonov A. O. and Karchevskiy E. M. Projection methods for computation of spectral characteristics of weakly guiding optical waveguides. Proceedings of the International Conference Days on Diffraction 2013, St. Petersburg, Russian Federation, pp. 131-135, 2013.

[50] Karchevskii E. M., Spiridonov A. O., Repina A. I., and Beilina L. Reconstruction of Dielectric Constants of Core and Cladding of Optical Fibers Using Propagation Constants Measurements. Physics Research International, Vol. 2014, Article ID 253435, 2014. 
[51] Beilina L. and Karchevskii E. The layer-stripping algorithm for reconstruction of dielectrics in an optical fiber. Inverse problems and applications, Springer Proceedings in Mathematics and Statistics, 120, pp. 125$134,2015$.

[52] Karchevskii E., Spiridonov A., and Beilina L. Determination of permittivity from propagation constant measurements in optical fibers. Inverse problems and applications, Springer Proceedings in Mathematics and Statistics, Vol. 120, pp. 55-65, 2015.

[53] Karchevskii E. M., Beilina L., Spiridonov A. O. and Repina A. I. Reconstruction of dielectric constants of multi-layered optical fibers using propagation constants measurements. Applied and computational mathematics, vol 15(3), pp. 346-358, 2016.

[54] Rezgui H. and Choutri A. An inverse eigenvalue problem. application: Graded-index optical fibers. Opt. Quant. Electron., vol. 49(10), 2017.

[55] Sladen F. M. E., Payne D. N., and Adams M. J. Determination of optical fiber refractive index profiles by a near-field scanning technique. Applied Physics Letters, 1976.

[56] Marcuse D. Theory of dielectric optical waveguides, Second edition. Academic Press, New-York, 1991.

[57] Snyder A. W. and Love J. D. Optical waveguide theory. Chapman \& Hall, London, 1983.

[58] Vassalo C. Théorie des guides d'ondes électromagnétiques, Tomes $1 \&$ 2. Eyrolls et CNET-ENST, Paris, 1985.

[59] Bonnet A. S. and Djellouli R. High-frequency asymptotic of guided modes in optical fibers. IMA J. Appl. Math. 52, (1994), pp. 271-287.

[60] Ishigure T., Endo H., Ohdoko K., Takahashi K., and Koike Y. Modal Bandwidth Enhancement in a Plastic Optical Fiber by W-Refractive Index Profile. Journal of lightwave Technology, 23, No. 4, (2005), pp.17541762 .

[61] Carleman T. Sur un Problème d'Unicité pour les Systèmes d'Équations aux Dérivées Partielles à Deux Variables Indépendantes. Ark. Mat. Astr. Fys. B 26 (17), (1939), pp. 1-9.

[62] Hörmander L. Linear Partial Differential Operators. Springer Verlag, Berlin-Heidelberg-New York, 1969. 
[63] Bamberger A., Bonnet A. S., and Djellouli R. Calcul des modes guidés d'une fibre optique. Première partie: différentes formulations mathématiques du problème. Rapport Interne, 142, Ecole Polytechnique, 1985.

[64] Bamberger A. and Bonnet A. S. Mathematical analysis of the guided modes of an optical fiber. SIAM J. Math. Anal., 21(6), pp. 1487-1510, 1990.

[65] Engl H. W., Hanke M., and Nebauer A. Regularization of inverse problems. Kluwer Academic Publisher, Dordrecht, 1996.

[66] Gilyazov S. F. and Goldman N. L. Regularization of ill-posed problems by iterative methods. Kluwer Academic Publisher, Dordrecht, 2000.

[67] Djellouli R. Inverse acoustic problems. In: Computational Methods for Acoustic Problems, Saxe-Coburg Publications, 2008.

[68] Tikhonov A. N. Regularization of incorrectly posed problems. Soviet Math. Doklady, 4, (1963), pp. 1624-1627.

[69] Tikhonov A. N. and Arsenin V. Y. Solutions of Ill-Posed Problems. Winston, New York, 1977.

[70] Morozov V. A. On the solution of functional equations by the method of the regularization. Soviet Math. Doklady, 7, (1966), pp. 414-417 (English translation).

[71] Morozov V. A. Choice of parameter for the solution of functional equations by the regularization method. Soviet Math. Doklady, 8, (1967), pp. 1000-1003 (English translation).

[72] Wahba G. Spline models for observational data. SIAM, 1990.

[73] Hansen P. C. and O'Leary D. The use of the l-curve in the regularization of discrete ill-posed problems. SIAM J. Sci. Comput., 14, (1993), pp. $1487-1503$.

[74] Djellouli R., Bekkey C., Choutri A. and Rezgui H. A Local Boundary Condition Coupled to a Finite Element Method to Compute Guided Modes of Optical Fibres under the Weak Guidance Assumptions. Math. Meth. Appl. Sci., 23, (2000), pp. 1551-1583.

[75] Barucq H.,Bekkey C., and Djellouli R. Construction of local boundary conditions for an eigenvalue problem. Application to optical waveguide problems. Journal of Computational Physics 193, (2004), pp. 666-696. 
[76] Raviart P. A. and Thomas J. M. Introduction à l'analyse numérique des équations aux dérivées partielles. Masson, Paris, 1983.

[77] Ciarlet P. G. The Finite Element Method of Elliptic Problems. NorthHolland, Amsterdam, 1978.

[78] Lehoucq R.B., Sorensen D.C., and Yang C. ARPACK Users guide: Solutions for large scale eigenvalue problems by implicitly restarted Arnoldi methods. Software Environ. Tools, 6, 1997.

[79] Saad Y. Numerical Methods for Large Eigenvalue Problems. Manchester University Press, New York, 1992.

[80] Wilkinson J. H. The Algebraic Eigenvalue Problem. Clarendon Press, Oxford, UK, 1965.

[81] Abramowitz A., Stugun I. A. Handbook of Mathematical Functions. Dover, New York, 1964.

[82] Atkinson K. E. An introduction to Numerical Analysis. Second Edition, John Wiley \& Sons, 1989.

[83] Golub G. H., Van Loan C. F., Matrix Computations, The Johns Hopkins University Press, Baltimore and London, 1996.

[84] Gloge D. Weakly Guiding Fibers. Appl. Opt.,Vol.10, pp. 2252-2258, 1971.

[85] Sharma E., Goyal I., and Ghatak A. Calculation of cutoff frequencies in optical fibers for arbitrary profiles using the matrix method. IEEE Journal of Quantum Electronics, Vol. 17(12), 1981. 